\title{
Assessing transport related social exclusion using a Capabilities Approach to accessibility framework: A dynamic Bayesian network approach
}

1 Abstract
Accessibility is considered to be a valuable concept that can be used to generate insights on issues related to social exclusion due to limited access to transport options. Recently, researchers have attempted to link accessibility with popular theories of social justice such as Amartya Sen's Capabilities Approach (CA). Such studies have set the theoretical foundations on the way accessibility can be expressed through the CA, however, attempts to operationalise this approach remain fragmented and predominantly qualitative in nature. In this study, a novel framework of expressing accessibility at the level of an individual is proposed, based on the basic elements of the CA. In particular, dynamic Bayesian networks are used to express the causal relationship between capabilities, functionings, personal and environmental characteristics. This is done by introducing informative Dirichlet prior distributions constructed using data from traditional mobility surveys, modelling the transition probabilities with data related to place based characteristics and defining an observation model from unlabelled mobility data and places of interest (POI). We demonstrate the usefulness of the proposed framework by assessing the equality levels and their link to transport related social exclusion of different population groups in London, using unlabelled, service provider generated mobility data.

\section{Introduction}

The concept of accessibility has been the focus of different disciplines such as geography, urban planning and transport planning for some time. The wide adoption of the term resulted in different definitions commonly encountered throughout literature: A very early definition originates from 36 Hansen (1959) who defined accessibility as a po- ${ }_{37}$ tential of interaction between destinations. Within 38 transport economics Ben-Akiva (1979) defined ac- 39 cessibility based on the benefits provided by the 40 interaction between transport and land use. In 41 transport geography Geurs \& Van Wee (2004) de- 42 fined accessibility as the extent to which transport 43 and land-use systems enable individuals or groups $\quad 44$ of individuals to reach activities or destinations by $\quad 45$ means of transport modes. $\quad 46$

When the focus of the studies is the connection 47 between accessibility and social processes causing disadvantage, such as transport related social exclusion, the term accessibility is generally viewed as a fundamental property of individuals to participate in different activities within civil society (Burns 1980, Preston \& Rajé 2007) and refers to the extent to which a person is able to reach a range of destinations that facilitate different social, leisure and employment activities considered to be normal for their society (Evans 2009, Nutley 1998). This ability takes the wider urban environment characteristics into consideration, such as transport provision (buses, trains etc.) and environmental characteristics as well as individual preferences and capabilities (Farrington 2007, Kwan 2013). Related to this, Church et al. (2000) identified seven distinct factors that could reduce access to opportunities, covering aspects such as physical characteristics of an individual (eg. mobility difficulties, impairments etc.), geographical and place based characteristics, time based restrictions as well as economic and societal factors. It is important to note that these factors tend not to appear in isolation, and coexisting factors are more 
likely to increase the risk of transport related social exclusion.

Although the above description of accessibility overlaps with the notion of mobility, it also highlights some key concepts that tend to be overlooked by thinking only in terms of mobility. Traditionally, in transportation planning and engineering, individual mobility refers to the resources and characteristics of individuals (financial status, age, access to a car etc.) that enable a person to move from place to place (Tyler 2006). However, increased mobility does not necessarily result in increased accessibility. For example, a person can be thoroughly mobile and still experience barriers when attempting to reach an activity. Besides physical and geographical, these barriers could be of a social nature such as social discrimination or fear of crime. (Church et al. 2000, Evans 2009). In any case, the mobility component is implicitly included in the definition of accessibility as given above.

In terms of accessibility measurement, there is a rich history of different numerical approaches, depending on the geographical scale and target group of an accessibility assessment. One fundamental categorisation given by Miller (2005) is place-based measures and people-based measures. The former focuses on place based and spatial separation concepts while the latter focuses on individual accessibility/mobility patterns. While measures from both categories have been used to investigate issues of transport related social exclusion, the choice of measure can produce dramatically different results. These range from overestimating equity of access to different urban services, as is the case of placebased measures, to producing more conservative, but oversensitive results (Neutens et al. 2010).

Recently, there has been an interest in using Amartya Sen's Capabilities Approach (CA) to express accessibility using theories of social justice (Hananel \& Berechman 2016). This framework can then be used for investigating equity issues in transport. Apart from providing decision makers with a framework for considering equality in transport provision, the components of the approach provide the flexibility to express complex concepts, such as accessibility, through a causal structure (Pereira et al. 2017, Hananel \& Berechman 2016
Beyazit 2011). In this study, a new numerical framework is presented for evaluating individual accessibility, using the Capabilities Approach. The implementation is based on dynamic Bayesian networks, and provides both inferential and computational intelligence capabilities using unlabelled mobility data. Furthermore, this study links the discovered accessibility patterns with socio-economic qualitative attributes at an individual level.

Contrary to previous articles that have used this approach within qualitative case studies, we demonstrate its applicability to investigating equality levels in accessibility using a combination of machine generated service provider data, in particular London's Automatic Fare Collection (AFC) system, and further socio-economic data. The paper is organised as follows. In section 2, the link between accessibility and transport social exclusion is briefly discussed as well as the placement of accessibility within social justice theories. Section 3 introduces the Capabilities Approach to accessibility model and provides implementation details by specifying the elements of the Capabilities set and how these relate to the observed functionings. Finally section 4 provides the results and sections 5 and 6 provide the discussion and conclusions, respectively.

\section{Research Background \\ 2.1 Accessibility and transport related social exclusion}

The link between transport disadvantage and issues such as social exclusion, well-being and discussions around issues of equity and equality has been recognised since the 1960's. Fairly recently however, this discussion has been extended to recognise the fundamental role of accessibility in such issues (Pereira et al. 2017, Lucas 2012, Casas 2007). According to a widely cited definition by Kenyon (2003), transport related social exclusion is a process by which individuals are prevented from participating in different aspects of a social life in a community. This may be because of reduced accessibility to opportunities, services and social networks or due to insufficient mobility in a society. Such a process leads to decreased levels of wellbeing particularly for vulnerable population groups (Currie et al. 2010). 
A social exclusion approach to transport disadvantage puts the focus on the outcomes of transport deprivation (Titheridge et al. 2014), however, it is important to notice that this concept emphasizes both the causal factors that lead to such a condition and the interactions between them (Lucas 2012). Such factors include characteristics that lie with the individual, characteristics of the local area as well as wider economic societal and governance factors. The lack of available transport options or inability to use them, together with disadvantaged personal status reduces the ability of an individual to reach different opportunities, causing lack of accessibility, which is in turn manifested as social exclusion.

Along the same line of thought, Preston \& Rajé (2007) argue that the effects of social exclusion are not due to a lack of social opportunities, but because of a lack of access to those opportunities. According to the authors, addressing social exclusion requires extending the knowledge of person/place interaction beyond transport geography and into the domain of social-spatial research. Approaching accessibility from this angle, Farrington \& Farrington (2005) redefine the terms used to describe accessibility: Opportunities become more than locations on a map. They are potentials for achieving an individual's needs, wants, aspirations and desires. Reaching opportunities becomes more than a function of space, as an individual won't necessarily be able to participate in the activities associated with each destination (Pereira et al. 2017).

At this point, it should be noted that case studies seeking to quantify transport disadvantage or transport related social exclusion 11 rarely adopt the above described definition of accessibility in its entirety. Instead, existing accessibility indicators covering aspects of the above definition are used (Kamruzzaman et al. 2016, Pyrialakou et al. 2016). For example, Preston \& Rajé (2007) used a gravity and utility based accessibility indicator to identify areas of different levels of mobility/accessibility at an aggregated level. Wu \& Hine (2003) used a contour based accessibility approach to identify transport disadvantage in households living in ar-

\footnotetext{
${ }^{1}$ Although transport disadvantage and transport related social exclusion are different concepts, the indicators used in case studies are often identical (Kamruzzaman et al. 2016)
}

eas with limited transport coverage. Commonly 212 mentioned reasons for this are the lack of data 213 availability, the convenience of using already estab- 214 lished models as well as the need to communicate 215 the findings in a familiar manner to policy makers. 216

However, attempting to approach issues of so- 217 cial equity using existing accessibility measures can 218 be problematic. Using different frameworks of so- 219 cial theory, Martens \& Golub (2012) examined how 220 different accessibility measures could perform in 221 terms of equity. They argued that neither place 222 based frameworks such as distance and infrastruc- 223 ture based measures, nor people based frameworks, 224 such as space-time and utility based measures, are 225 suited to address issues of social justice in trans- 226 port. The former is mainly mobility oriented, in 227 that it focuses only on the ability of a person to 228 travel in space but not actually to what he/she 229 can do with the opportunities offered at a destina- 230 tion. For example, there might be fully accessible 231 buses for people with disability, but this is not of 232 much use if the destinations lack accessible facili- 233 ties. People based frameworks on the other hand, 234 by looking only at actual travelling patterns, con- 235 ceal a basic equity argument (Sen et al. 1990): a ${ }_{236}$ person might adjust his/her expectations to deal ${ }_{237}$ with the conditions at hand. For example, a dis- 238 abled person might manage to get to work by trav- 239 elling twice the amount of time compared to a 240 non-disabled person, but that doesn't mean that 241 the person should not opt for better transportation 242 conditions. These examples highlight that an indi- 243 vidualistic, people based approach to accessibility 244 needs to be combined with qualitative appraisals 245 that could aid towards a deeper understanding of 246 inequalities.

Finally, it should also be mentioned that accessibility is only one way of quantifying transport related social exclusion, albeit the most holistic one. Other methods include structured questionnaires and basic statistical analysis (Delbosc \& Currie 2011), outcome based analysis such as measurement of individual activity spaces (Schönfelder \& Axhausen 2003), deprivation based measures (Noble et al. 2007), mobility based measures (Dodson et al. 2006) and structural equation models (Golob \& McNally 1997).

2

3

4

16

7

8

19
220

21

22

23

4

225

226

27

228

229

230

32

33

234

235

236

237

238

239

240

241

242

243

244




\subsection{The Capabilities Approach and ac- cessibility}

The CA was first introduced by the philosopher and economist Amartya Sen in the 1980's (Sen et al. 1990), and was originally developed as an alternative to the predominant utilitarian way of viewing notions such as quality of life and wellbeing in welfare economics. It's success as a theory of social justice has led to the creation of the $\mathrm{Hu}$ man Development Index by the United Nations Development Programme for the purposes of ranking countries by the level of well-being. In essence, the CA describes the ability of an individual to function given the set of practical opportunities that are available to them (Sen et al. 1990). Contrary to Rawl's egalitarian approach (Rawls 2009) where the emphasis is on the primary goods, the CA focuses on human capabilities which result from a combination of personal abilities, and the wider environment (Pereira et al. 2017).

The CA can be perceived as a normative evaluation concept, aiming at promoting public policies towards improvement of the abilities of individuals to function as opposed to just describing the problem. This allows for the relative assessment of different policy proposals and the effect that those will have on a person's well-being (Alkire|2008). As accessibility has been traditionally used as a concept that can push towards policy changes (Pirie 1981) the Capabilities Approach seem to fit in that framework. Viewing accessibility within this context encompasses not only the ability of individuals to move so that they can conduct the activities they value or have reason to value, but also includes all the policies that enable people to do so (Pereira et al. 2017). Two notions are central in this theory: capabilities and functionings:

- Capabilities: These refer to the practical opportunities available and are the combinations of beings and doings that a person can achieve.

- Functionings: These refer to the various things a person may value doing and being (Sen 2014) and are usually observed (realised) representing what an individual actually achieves.

In accessibility terms, functioning can be un- derstood as the realisation of day-to-day activities (e.g. shopping, getting to work etc.). The practical opportunities constitute the capabilities that each person has to complete the activity. Although the capability set is not directly observable, it can be derived from a set of functioning vectors from which the person has the freedom to choose (Mitra 2006). In this reading, the Capabilities Approach can be used to capture elements of social freedom (the ability to achieve various functions and realise one's potential), welfare (the capability to achieve these functions) and equity (Hananel \& Berechman 2016).

Within the CA, the notion of functioning vectors refers to all factors that shape the capabilities set. The scope of functioning vectors can be very broad and can include different elements such as an individual's characteristics (e.g. age, income, impairment etc.), characteristics of the environment (e.g. social, physical, cultural etc.) or commodities (e.g. availability of public transport modes).

\subsection{The capabilities approach in trans- portation literature}

Literature on applications of the $\mathrm{CA}$ in transportation is sparse, however, it clearly sets the scene on how it can be used to assess accessibility.

Hananel \& Berechman (2016) argue that the first step towards translating the CA in the transportation domain is to define what is meant by capabilities. In their view, a combination of the extent of mobility and access to opportunities for individual population groups, especially the disadvantaged ones, could be considered as good candidates for capabilities. These capabilities should reflect the minimum conditions that allow the least advantaged groups to benefit from any transportation interventions. Thus, the functioning vectors may include measures such as the maximum allowable travel time, travel distance or travel expenses for all residents in the area of influence, focusing on the more disadvantaged. The authors conclude that the capabilities and functioning vectors should not be viewed independent from one another, but recognise and address the interactions between them.

In another study, Beyazit (2011) juxtaposed the 
core elements of the CA with concepts in transport research. In their analysis, functionings refer to the wider definition of accessibility as it has been described in the section 1. Particularly, the transport system constitutes the goods, while the provision of access to ones needs and wants is the functioning of the transport system. Travelling for leisure could be one of these functionings, as is travelling for social interaction. The capabilities then refer to the mobility element that enables people to move from one location to another physically, socially and financially, within a society and across societies. In this way, people possess a capabilities set which translates into an opportunities set of achievable functionings, from which they are free to chose. Manifestations of these choices could be the traveling mode or modes, the choice of locations, the reason to travel and the choice of travel time.

Hickman et al. (2017) interpretation of functionings and capabilities within the transport context is similar to that of the authors above. In their view, the functionings represent what a person actually does and how. The realised functioning element is represented by the actual travel behaviour and participation in activities and as such, it is easier to measure. Measurement of capabilities on the other hand is more challenging. The authors propose an individual based accessibility definition that encompasses, alongside physical accessibility, issues such as the type of available infrastructure, land use, social and cultural norms and individual characteristics. The defined capabilities set is specific to each individual and reflects the freedom to choose from different potential functionings. However, this doesn't mean that two persons with similar functionings have the same capabilities. For example, a person with higher income may choose to have a similar mobility level to a person of a lower income by choosing not to own or use a car.

This distinction between functionings and capabilities is beneficial in that it helps towards understanding why improvements in certain levels of accessibility (such as improvement in levels of public transport, new cycling infrastructure etc.) might not lead to improvement in the overall accessibility.

Pereira et al. (2017) proposes framing accessibility in terms of combined capabilities, having two separable but interacting components. This first one relates to a person's capability to access and use the transportation system, which depends on the interplay between personal and external factors. Personal factors may be individual characteristics such as physical and mental health, accumulated experience and financial resources. External factors may be the social environment as well as the transport system's design, price level information or availability. The second component refers to the more macroscopic view of accessibility which is related to the interaction between the transportation system and land-use patterns, and how this interaction acts as an enabler towards the expansion of capabilities. This includes elements of the transportation network such as network coverage and connectivity, as well as the spatial distribution of activities.

Tyler (2006) approached accessibility through the CA following a more microscopic view. In this setting, capabilities are perceived as the combination between the individual abilities of a person, and the capabilities the environment provides. To recite the author's example, the physical infrastructure might require someone to be able to step up $30 \mathrm{~cm}$ to participate in an activity. If the person is not able to provide this capability based on the individual characteristics (eg. wheelchair user or the elderly), then participation in the activity is not possible. Therefore, there is an interaction between what an individual can offer and what the environment can provide.

Looking at the mobility component of accessibility for elderly people, Ryan et al. (2015), approached capabilities as the outcome of an individual's mobility resources. In this sense, the potential of an individual to use public transport constitutes an element of the capabilities set. Functionings are chosen by an individual from the elements of the capabilities set, which could be all the different transportation options. The definition of realised functionings as actual behaviour is in line with the previously described studies.

In a study to identify Minimum Income Standards for transport use within rural communities, (Smith et al. 2012) used the CA to place income in the wider notion of well-being. Income however, is only one of the factors that affect people's capabilities to function. As a result, the authors extended 
the definition of minimum income to refer to all the goods, services, opportunities and choices to participate in society.

In another study (Hickman et al.|2017), used the notions of desired and actual transportation situation to distinguish between capabilities and functionings. Particular elements of the capabilities set included, among others, proximity to transportation access points, air quality levels, levels of security, levels of enjoyment when travelling, levels of accessibility to employment, availability of transportation modes, commuting time and transport costs.

Other authors (Orr 2010), proposed defining the capabilities set by focusing on activities, both realised and potential. Once these are identified, the individual capabilities required to achieve these can be mapped out (eg. access to sufficient income). This approach is framed in terms of evaluation of different transportation interventions aiming at minimising transport disadvantage and social exclusion for elderly and disabled people. They proposed to break down an activity into individual tasks and assess each task individually. For example, the activity 'going to a shop' has a set of necessary tasks embedded, one which could be 'taking the bus'. Specific barriers can then be associated with particular tasks, such as 'fear of crime walking to the bus stop'. In contrast to the above mentioned case studies, this approach to defining and measuring the capabilities set is inherently data driven.

Table 1 below summarises the way different authors approached definitions of the capabilities set and functionings as well as input variables.

Judging from the reviewed studies, the CA has been applied to a wide range of social issues in transport, ranging from investigating the impact of specific transport interventions to evaluating transport related social exclusion. In nearly all cases, the studies were based on empirical findings within a specific geographical context while the focus was on disadvantaged groups (eg. low income people, elderly, slum dwellers etc.) and within a comparative evaluation framework. A considerable proportion of the reviewed studies were qualitative, in line with the body of literature covering social aspects of transport (Lucas \& Porter 2016). The ones that were more quantitatively oriented used statistical 496 tools such as Structural Equation Models, Prin- 497 cipal Component Analysis, logistic regression etc. 498 This suggests that there is currently no consensus 499 among researchers on how to quantitatively opera- 500 tionalise the CA for issues related to transport and 501 social aspects. 


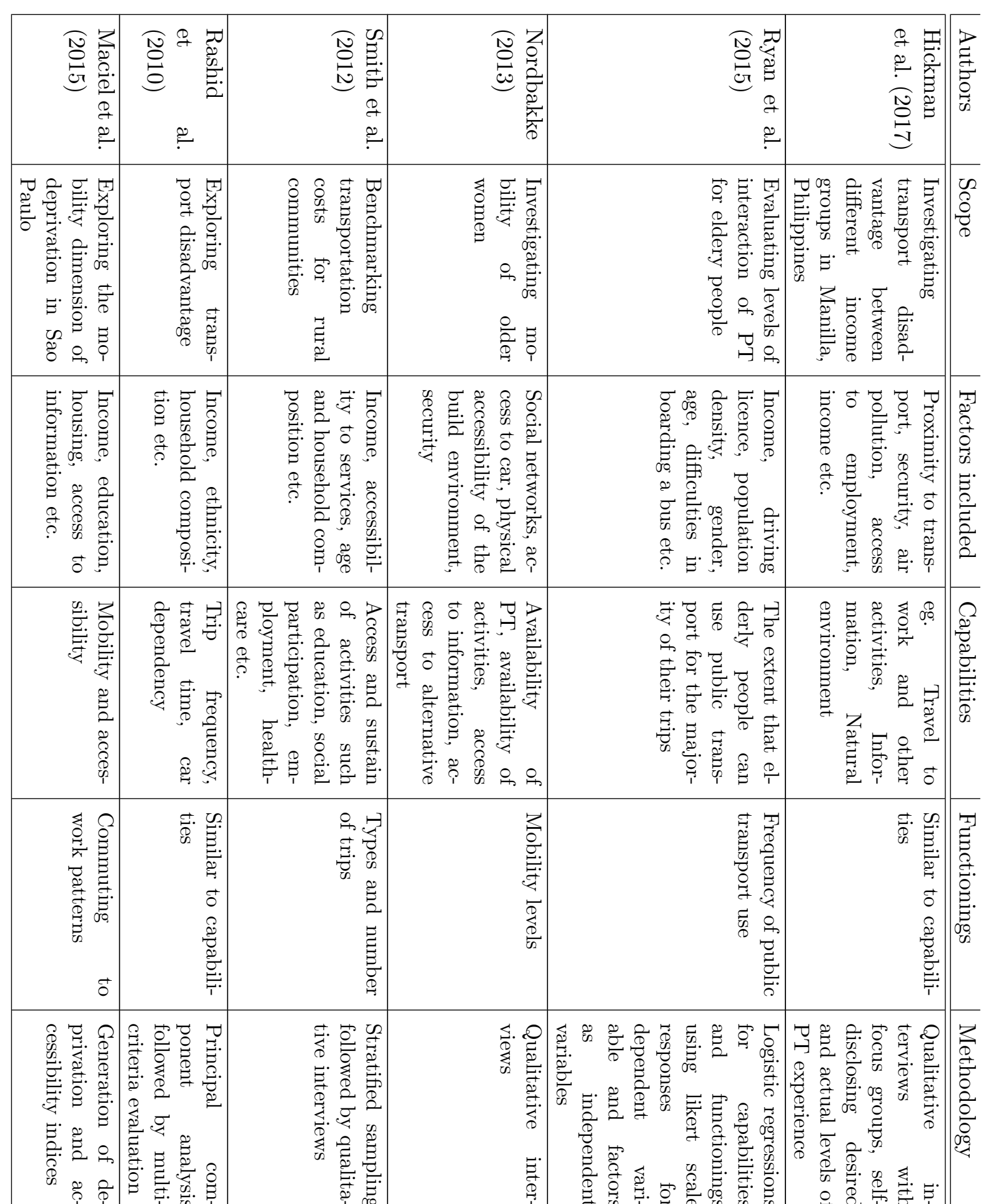




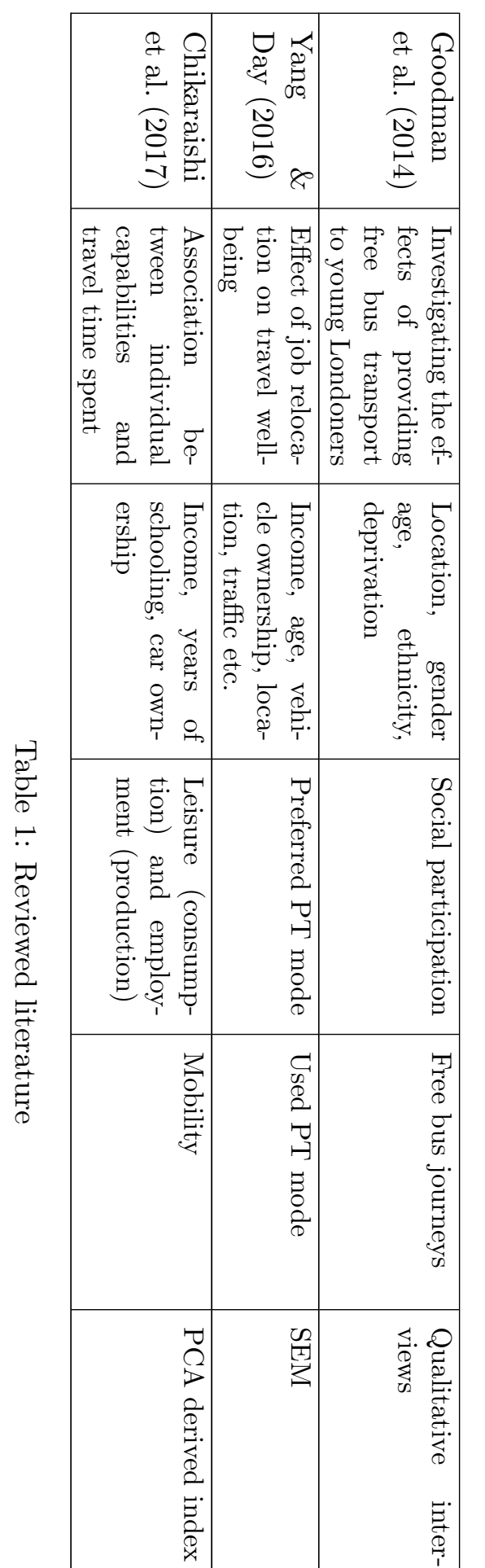


In terms of the link between the Capabilities Approach and accessibility, three common themes have been identified in all reviewed studies:

- Capabilities represent the potential of an individual to reach and engage with opportunities. Realised functionings represent the observed behaviour of the above. Both of the terms are in line with the general definition of accessibility as set out in section 1 .

- The focus of the CA is the individual and in this sense in line with person-based accessibility. Moreover, it takes into consideration the influence of internal and external factors that shape the individual capabilities set.

- The capabilities set is not static but in constant interaction with the components that shape it and the realised behaviour expressed by the actual functionings. The evolving nature of the capabilities set extends both spatially and temporally, in the sense that is modified based on location and time.

Moreover, the case studies emphasize the causal structure between the factors that shape the capabilities, the capabilities themselves and the functionings. This causal structure appears to be hierarchical in nature, with the functionings appearing at the bottom of the hierarchy and the factors appearing at the top.

The definition of the elements included in the capabilities set and the corresponding functionings is used interchangeably for some studies. This is not uncommon and has been identified in applications of the CA to other social aspects beyond transport (such as quality of life) (Robeyns 2005). Reasons for this can be traced in the definition of functionings as enablers to achieve the defined Capabilities, but also the close relationship between transport concepts such as mobility and accessibility (for example, mobility can be considered both a functioning (using the bus) and a capability (ability to move) (Chikaraishi 2017). In all cases, however, there is a distinction between what is measured (functionings) and hypothesis to be tested (capabilities).
On the other hand, there exists a general con- 547 sensus on the factors influencing the capabilities 548 set. This includes either focusing on the socioe- 549 conomic characteristics of an individual, the wider 550 environment (both physical and social) or both. In 551 line with social exclusion definition as provided by 552 the Social Exclusion Unit (Social Exclusion Unit 553 2003), sociodemographic variables such as income, 554 age and gender are all defining factors that influ- $\quad 555$ ence accessibility and have been included in the 556 majority of the studies. Variables of the wider so- 557 cial environment such as deprivation, although not 558 explicitly accounted for, have been taken into ac- 559 count during the design phase of most of the re- 560 viewed studies. Physical characteristics such as 561 distance to amenities, density of public transport 562 etc. have also been adopted as important factors 563 that shape the capabilities set by the majority of 564 the studies.

Finally, in spite of the advantages of passively generated mobility data from transport service providers, namely larger samples, regular update rate, low cost and the potential for longitudinal studies (Pelletier et al. 2011, Bagchi \& White 2005), none of the reviewed literature has explored their potential to extract quantifiable evidence of social exclusion and transport disadvantage. This is true within the accessibility literature in general (Anda et al. 2017) and the CA particular. This is largely due to the unlabelled nature of such datasets, requiring an additional step to infer activity types at a destination.

\section{A Capabilities Approach to ac- cessibility framework}

\subsection{Conceptual framework}

The different concepts of the CA and the way they are linked are shown in Fig. 1 (Mitra 2006. Beyazit 2011). At an observable level, one encounters the functionings of an individual. Within an accessibility setting, this node is referring to the realised activities as well as the realised transportation modes used to reach those activities. Moving one level up the hierarchy there exist the latent set of capabilities which form the choice set of an individual. These are all the potential opportunities an individual could choose. In this setting, 48 549 0 51 52 53

54

55

56

.

58

59

560

561

562

63

564


a realisation of a chosen element of the capabilities set leads to an observed functioning. This in turn is influenced by personal, environmental and social characteristics as well as the commodities a person has in his/her possession. All variables of this representation are expressed through stochastic quantities which aim to quantify uncertainty from incomplete knowledge about the state of variables, as is the case of capabilities, or from noisy and erroneous measurements as is the case of functionings.

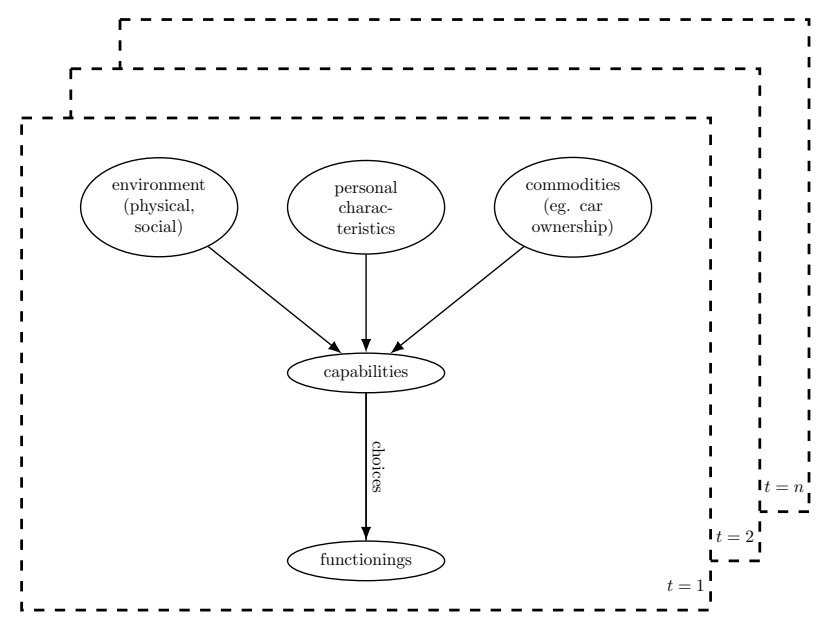

Figure 1: The CA (adopted from Mitra (2006))

The process is relevant for each individual and takes place in space and time during the act of reaching opportunities. In this setting, the capabilities set is changing depending of the characteristics of the environment that exist in each location at a particular point in time $(t=1 \ldots n)$. This representation imposes a structure on accessibility through the use of a directed graph where the nodes represent the components of the CA and the edges the relationship between them. The graph is acyclic, in the sense that no closed loops appear between the nodes. This allows information to flow from the top level to the bottom level nodes. The whole process should not be independent between subsequent time steps but should capture the dynamic evolution of capabilities in time.

In terms of mathematical implementation, the above described conceptual framework can be implemented through a Bayesian network structure, the details of which is described in subsequent sections. Bayesian networks have been success- fully used within transportation research for a wide range of applications, ranging from transportation mode detection (Bantis \& Haworth 2017) to travel behaviour analysis (Daziano et al. 2013). In the context of this study, advantages of using Bayesian networks can be summarised by the requirement of expressing accessibility through the causal structure of $\mathrm{CA}$ at an individual level, while at the same time providing inferential abilities from unlabelled mobility data. In addition, through the use of the posterior quantities for the model's nodes, Bayesian networks can represent uncertainty as a function of the different configurations of the states of all other variables in the model. Other approaches commonly used in the literature to represent casual relationships, such as SEMs, become unsuitable in the context of unlabelled mobility data. This is because SEMs do not provide inferential capabilities to extract semantic information from low level data.

\subsection{Data}

For this study, individual mobility data from London's AFC system (referred to as Oyster card) were used to infer the potential activity types an individual is likely to perform as well as the transportation modes used. In the 8-week sample provided (late October - mid December 2013), the individual trajectories represent the locations of public transport access points an individual used throughout their trips, as well as the public transportation modes used (Bus, Rail, Tram). The potential activities at each location were represented using Ordnance Survey's Points of Interest (POI) dataset (Ordnance Survey 2012), bounded by a 20 minute walking distance isochrone area at each location. From the 10-fold classification scheme defined by OS (Ordnance Survey 2012), four were considered representative for non-workplace activities (Accommodation, eating and drinking, Outdoors and recreation, Education and health, Retail) and one for employment activities (Commercial services).

Personal socio-demographic characteristics for each individual were obtained from a travel diary survey (London Travel Demand Survey, LTDS). LTDS is a continuous household survey aimed at probing London's public transport customers' so- 
ciodemographic background and patterns of transport, with a geographic coverage extending up to outer Greater London but within the M25 boundary.

During the 2011/2012 LTDS survey, respondents were asked if they were willing to provide their Oyster card unique ID for Transport for London (TfL) to undertake further analysis of their travel. Since then, the relevant data has been stored by TfL's Customer Experience department from midJune 2011 to March 2014. The Oyster card sample provided for this study, overlapped with that of the LTDS sample for the period of October/midDecember 2013. The trajectories were linked to sociodemographic characteristics by means of a the Oyster card unique ID present in both datasets.

Differentiation between employment and nonemployment activity types within an individual's trajectory was done by utilising the information contained in the combined LTDS/Oyster card dataset and duration of stay ${ }^{2}$, In particular, the distributions of duration of stay for individual daily journeys were plotted and examined for each employment status. The probability of an activity belonging to Employment was then calculated using the probability density function of a logistic random variable.

$$
f(x ; \mu, \sigma)=\frac{e^{-\frac{\mu-x}{\sigma}}}{\sigma\left(1+e^{-\frac{\mu-x}{\sigma}}\right)^{2}}
$$

where $x$ is the duration in hours, $\mu$ the location parameter and $\sigma$ the standard deviation (scale). The parameters $\mu$ and $\sigma$ were adjusted to reflect different working assumptions as assessed empirically by the duration of stay distribution of Figure 10.

Equation 1 was then used to weight the OS activity type vector corresponding to Employment/Education activity types.

\footnotetext{
${ }^{2}$ In the context of this study, this was defined as the duration between individual trip segments, using only the records that are less likely to belong to an interchange trip
}

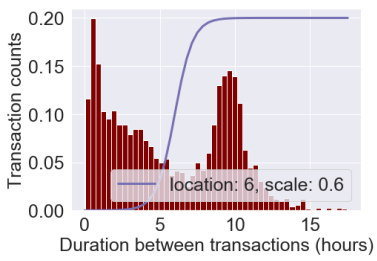

(a) Full-time paid employment

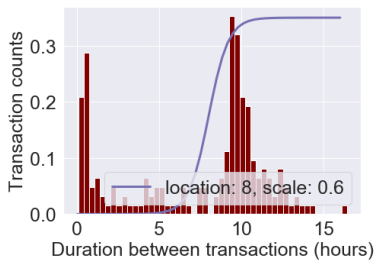

(c) Full-time selfemployment

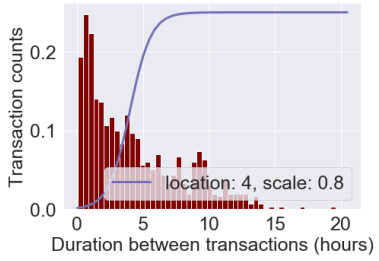

(b) Student/school pupil

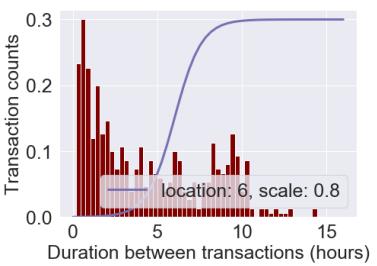

(d) Part-time paid employment
Figure 2: Distribution of duration between transactions for different employment types, with logistic cumulative distribution functions (CDF) overlayed. Note that the histograms were normalised and the CDFs were scaled accordingly.

In addition to the above described data, a num- 707 ber of other data sources were used to shape as- 708 sumptions about the influence of personal charac- 709 teristics and external environment in an individ- 710 ual's ability to reach activities using the public 711 transport. Table 2 provides a summary of the data 712 used in this study. 


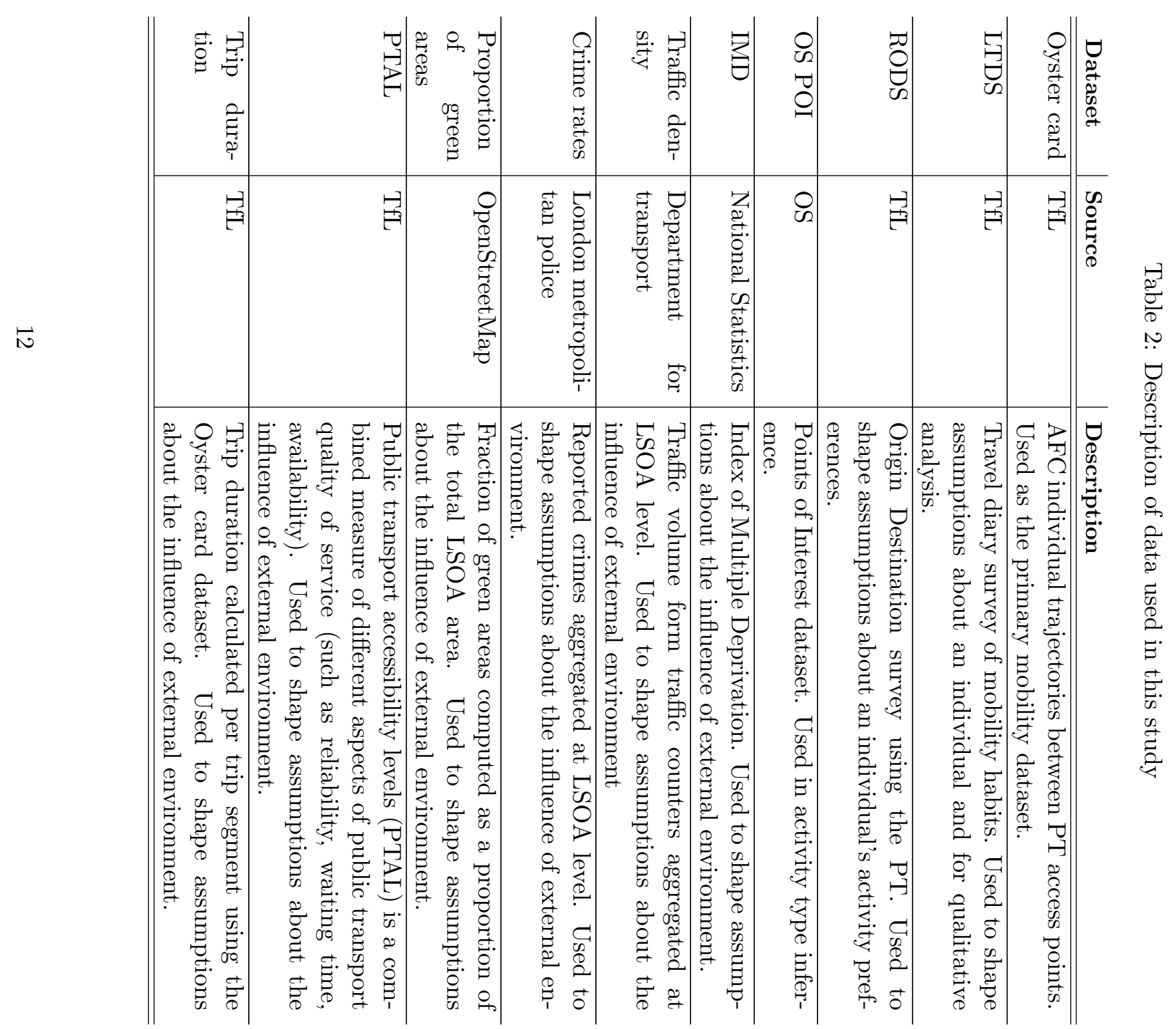


Using this information, the framework of comparison for this case study is based on three population groups: individuals having annual household income below $£ 15,000$, individuals $>60$ years of age and an unconstrained (base) population group. The choice of those population groups was based on two factors: the sample size of each group and past research providing evidence of population groups with significantly different accessibility levels compared to the majority of population (Páez et al. 2010, Hickman et al. 2017, Kamruzzaman et al. 2016, Titheridge et al. 2009). Deviations from equality will be assessed by analysing the elements of the capabilities set using the Theil's index. The results of this task can then identify gaps between the defined capability nodes that contribute to different levels of mobility using public transport and access to activity types.

Figure 3 below shows the geographic distribution of visited places for each population group.

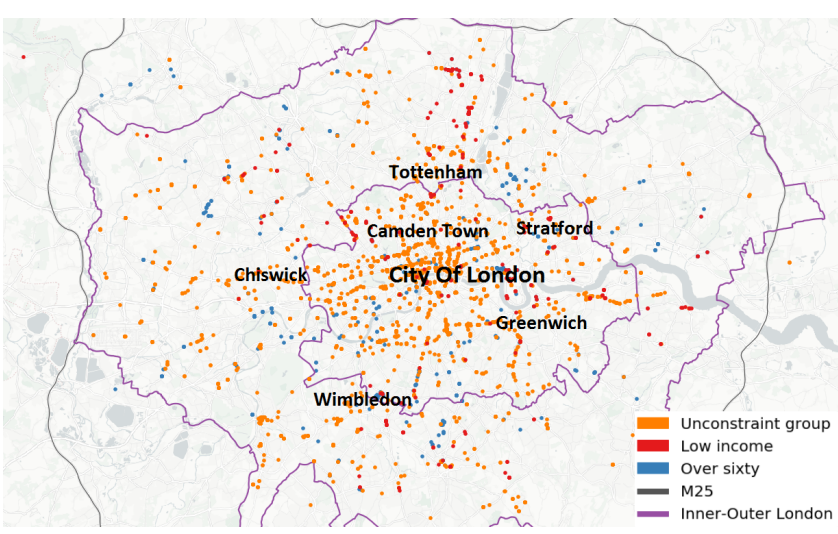

Figure 3: Visited places per population group.

As it can be seen, the majority of visited locations for the unconstrained and $>60$ years old population groups is concentrated within the boundaries of Inner London in general, and around the area of City of London in particular. This is not surprising since the majority of employment opportunities is located in this area. On the other hand, the geographical distribution of the low income population group appears to span radially from Inner London, with a significant concentration around Tottenham area.

\subsection{Model implementation}

In the specification of this case study, two dis- 747 tinct but interacting components of an individual's act of reaching opportunities are included:

- Ability to interact with the public transportation modes available

750

- Ability to interact with the destina- 752 tion/opportunities available

Following the graphical representation of Figure 1. a dynamic Bayesian network was defined, the nodes of which reflect the structure of Figure 1 The sections below describe the individual components of the model.

\subsubsection{Defining the Capabilities Set}

By definition, the set of capabilities should be constructed in a way that reflects an individual's choice to to realise their desired goals, as well as the potential opportunities an individual has to make those choices. According to Hananel \& Berechman (2016), an evaluation of the capabilities set should start by explicitly stating what these are. In the context of accessibility and adopting the definition of capabilities from Tyler (2006), these are framed around:

754

- the ability to engage with available opportu- 770 nities and

- the ability to use the public transport to do 772 so.

The first bullet point is related to the probability distribution of activity types bounded by the isochrone polygon, while the second is related to the probability distribution of using the different public transport modes at each access point in a trajectory.

In particular, this case study investigates the following elements:

- Potential accessibility to activities 782

- Potential mobility 783

- Potential accessibility and potential mobility 784 dynamics 
Potential accessibility to activities This element of the capabilities set describes the potential range of activity types that are reachable from a public transport access point. In the model specification of this case study, the set of potential activities is represented as a sequence of latent (unobserved) stochastic variables which are inferred by the propensity to perform each activity category based on personal characteristics and the number of defined POI types within reach from the public transport access point.

The effect of personal characteristics to the likelihood of reaching an activity type was captured through a Dirichlet distribution, through with the concentration parameter vector $\alpha_{z}$. This represents the degree of prior belief that an individual is likely to be performing one activity type over the other. For example, it might be that prior studies point that arrival time between 11:0012:00 pm and age group $<21$ years old can be used to determine education over employment activity. This assumption can be represented by setting $\alpha_{\text {education }}>\alpha_{\text {employment }}$.

Smaller $\left(0<\alpha_{z}<1\right)$ values of $\alpha_{z}$ express less uncertainty in the preference of an activity type over the other. On the other hand, larger values $\left(\alpha_{z}>1\right)$ express more uncertainty about the preference of an individual for an activity type. In this study, the calculation of the shape of the prior was based on rolling origin destination survey (RODS) data. In particular, a multinomial regression model was fitted on the complete RODS dataset, and the predicted probabilities for each activity type were generated for each individual based on age, sex, disability status and arrival time. These were then used to construct the concentration parameter vector $\alpha_{z}$. The resulting predicted probabilities were then multiplied with Gamma distributed random variables with shape and rate parameters of the Gamma distribution $\mathrm{a}=\mathrm{b}=1$ to ensure that the concentration parameters follow an exponential distribution with rate proportional to the RODS predicted probabilities (Bantis \& Haworth 2019).

Potential mobility This element of capabilities set describes the potential of public transport use from the modes that are available. Similarly to potential accessibility, potential mobility is represented by a latent stochastic quantity that is inferred using the propensity of public transport use given an individual's sociodemographic characteristics and the distribution of transport modes from the Oyster card data.

Similarly to section 3.3.1, potential mobility was modelled using a categorical random variable over the Oyster card transportation mode types. This time, the propensity of an individual to use one mode over another was modelled using a multinomial regression on the LTDS dataset. In this case, the personal characteristics determining the choice of transportation mode were age, income, possession of travel pass, disability, car license, sex and ethnic group. The predicted probabilities were then recovered and used to shape the prior belief of using one mode over the others through the Dirichlet concentration parameters.

Potential accessibility and potential mobility dynamics In the context of this study, the dynamic evolution of the capabilities sets was captured using a set of transition matrices.

The underlying assumption that is made in this modelling step is that characteristics of the environment have a varying effect on the ability of an individual to reach an activity. For example, the levels of deprivation change from location to location, and this is expected to influence the choice of performing an activity type at a particular location. Similarly, the existence of more transportation options (expressed as increased levels of transport accessibility) are expected to influence the choice of transportation modes.

This assumption was represented by modelling the transition between subsequent transportation modes and activity types using a set of Multinomial logistic regressions on external covariates. The transition sequences for the inferred activities/transportation modes specified per each category were constructed as follows:

where $y_{i}=\operatorname{argmax}\left(z_{i}\right)$ in the case of activities, 873 and $y_{i}=m_{i}$ in the case of transportation mode. $\quad 874$

Essentially, the algorithm generates a transition 875 dataset from one category to another by looping 876 through the trajectory locations and identifying if a $\quad 877$ 


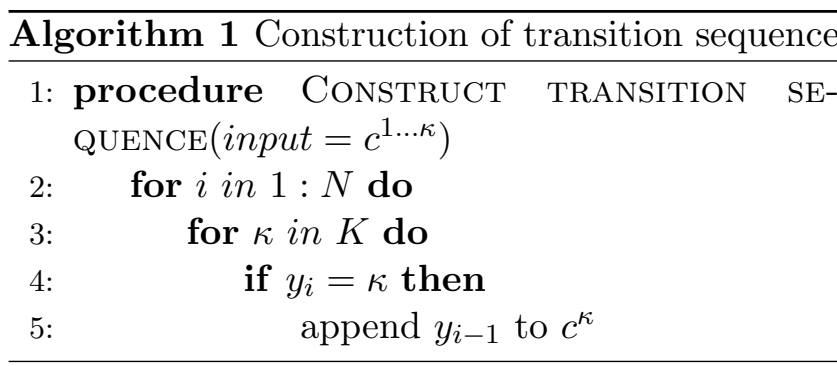

transition between location $n$ and location $n-1$ is related to activity types/transportation mode k. For example, consider a trajectory with transportation modes bus 1, bus $_{2}$, rail $_{3}$, bus $_{4}$. In this case, the row of the transition matrix corresponding to bus related transitions will be inferred using the sequence bus, rail as there is one bus/bus related transition (from $n=1$ to $n=2$ ) and one bus/rail related transition (from $n=2$ to $n=3$ )

The external covariates used were IMD, proportion of green spaces, traffic density, crime rate for the transitions between activity types and trip duration, PTAL for transitioning between different transportation modes (see table ??). These separate row regressions were organised in a row stochastic transition matrix and used in the calculation of the likelihood of potential accessibility/mobility. This resulted in two square row stochastic matrices, a $5 x 5$ matrix for the 5 activity categories $T_{z}$ and a $3 x 3$ matrix for the transportation modes $T_{m}$.

\subsubsection{Bringing it all together: Defining the structure of the model using Bayesian networks}

The CA to accessibility (CAA) model consists of two distinct but intertwined modules: 1) activity detection and modelling and; 2) mobility modelling. These are combined using a switch variable that activates the relevant module depending on whether an individual is using public transport or performing an activity. Figure 4 illustrates a graphical representation of the joint model.

Formally, the model is defined in equation 2 ;
Figure 4: Graphical representation of CAA model.

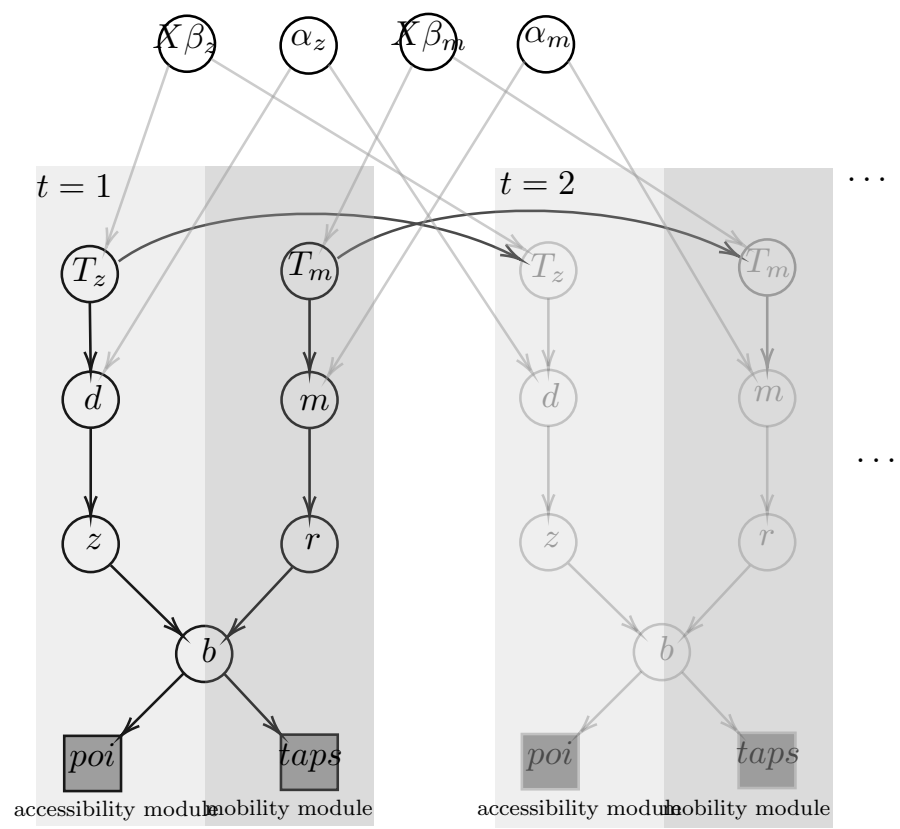

$$
\begin{gathered}
\beta \sim \operatorname{Normal}\left(0,10^{-3}\right) \\
s^{1 \ldots \kappa}=\frac{\exp \left(\alpha+\beta X_{n}\right)}{1+\sum_{\kappa=1}^{K-1} \exp \left(\alpha+\beta_{\kappa} X_{n}\right)} \\
c^{1 \ldots \kappa} \sim \operatorname{Categorical}\left(s^{1 \ldots \kappa}\right) \\
T=\left[\begin{array}{c}
s^{\kappa=1} \\
s^{\kappa=2} \\
\vdots \\
s^{\kappa=K}
\end{array}\right] \\
P\left(d_{n} \mid \alpha_{z}, T_{z}\right) \sim \operatorname{Dir}\left(\alpha_{z}, T_{\text {row }} \text { argmax }\left(d_{n-1}\right)\right) \\
P\left(z_{n} \mid d_{n}\right) \sim \operatorname{Mult}\left(\text { poi }_{n}, d_{n}\right)
\end{gathered}
$$

The mobility part of the inference is similar 911 to the above, with the exception that this time 912 the observation vector of transportation modes 913 is modelled through a categorical distribution 914 $P\left(r_{n} \mid m_{n}\right) \sim \operatorname{Cat}\left(m_{n}\right)$ with $P\left(m_{n} \mid \alpha_{m}\right) \sim \operatorname{Dir}\left(\alpha_{m}\right) . \quad 915$ In this case, the notion of functionings is more 916 straightforward as the Oyster card 'taps' are direct 917 observations on the actual choice of transportation 918 mode made by the individual. The node $b$ is a 919 stochastic variable acting as a switch that controls 920 which module is activated for inference (accessibil- 921 ity or mobility). It is assumed to follow a Bernoulli 922 distribution $P(b) \sim \operatorname{Bernoulli}(p)$, the probability ${ }_{923}$ 
of which is determined by the duration of stay relative to the cutoff value determined from the 95th percentile of the distribution of interchange times for bus and rail services (in the case of rail services, this was 15 minutes while for buses this was 36 minutes). For example, if the duration of stay between two subsequent bus trips is more than 36 minutes, then it is more likely that an activity is carried out at the stop (as opposed to being an interchange stop).

Finally, at the very bottom of the hierarchy of Figure 4, the square nodes represent the observed mobility and POI data used to infer the parent nodes. Table 3 below summarises the notation of the model:

Table 3: Description of variables of Figure 4

\begin{tabular}{l|l} 
Variable & Description \\
\hline \hline$p o i$ & $\begin{array}{l}\text { Count of POIs per activity within } \\
\text { the isochrone boundary }\end{array}$ \\
\hline$z$ & $\begin{array}{l}\text { Multinomial probability distribu- } \\
\text { tion of activities. }\end{array}$ \\
\hline$r$ & $\begin{array}{l}\text { Categorical probability distribution } \\
\text { of transportation modes. }\end{array}$ \\
\hline$m$ & Dirichlet distribution on $z$. \\
\hline$\alpha_{z, m}$ & $\begin{array}{l}\text { Ririchlet distribution on } r . \\
\text { tion parameter vectors for activity } \\
\text { types/transportation modes. }\end{array}$ \\
\hline$T_{z, m}$ & $\begin{array}{l}\text { Transition matrices for activity } \\
\text { types and transportation modes. }\end{array}$ \\
\hline$\beta_{z, m}$ & $\begin{array}{l}\text { Prior on external covariates } X ~ \\
\text { Normal }\left(0,10^{-3}\right) .\end{array}$ \\
\hline$b$ & $\begin{array}{l}\text { Bernoulli variable switching be- } \\
\text { tween modules during inference. }\end{array}$ \\
\hline
\end{tabular}

Inference on this the model was performed using Markov Chain Monte Carlo (MCMC) methods. A total of 20,000 sampling iterations were used, discarding the first 1,000 as non representative of the posterior quantities. The starting values of the stochastic variables were sampled from the prior distributions.

\subsection{A Thiel index based assessment 946 framework

Within the proposed framework of the CAA 948 model, two components of an individual's ability 949 to reach opportunities were identified and quan- 950 tified, given personal characteristics and external 951 factors: potential accessibility to different activity 952 types using the public transport, and potential mo- 953 bility of using the different transportation modes. 954 The first one is related to the concept of equality 955 of opportunities, while the second is related to is- 956 sues of transport disadvantage. The next step of 957 the analysis is to explore the relationship of the 958 components between individuals using the poste- 959 rior quantities as a basis of comparison.

Within the wider accessibility literature, the Theil index has been proposed as theoretically capable of quantifying accessibility related equity issues (Van Wee \& Geurs 2011) and has been applied as an equity evaluation tool for different case studies (Delafontaine et al. 2011, López et al. 2008).

The Theil index quantifies the actual entropy relative to the maximum entropy of the data and practically is a measure of difference between complete randomness and uncertainty and the observed state of the dataset configuration (equation 3):

$$
\begin{gathered}
S_{\text {Theil }}=\sum_{i=0}^{N}\left(\frac{x_{i}}{N \bar{x}} \ln \frac{N \bar{x}}{x_{i}}\right) \\
S_{\max }=\ln N \\
T=S_{\text {max }}-S_{\text {Theil }}
\end{gathered}
$$

where x is a vector of non-negative elements, $S_{\text {Theil }} \quad 967$ is the observed entropy and $S_{\max }$ is the theoretical 968 maximum entropy of the dataset.

It is interesting to observe that the above formulation is similar to Kullback-Leibler (KL) divergence (or relative entropy) if the vector $x$ is a valid discrete probability distribution, as is the case for the posterior distributions of model 4 , and $S_{\max }$ is the maximum entropy defined by the cardinality of the event set. KL-divergence is a commonly used probability divergence measure used to compare probability distributions within the context of applications in information theory (Cohen \& Kempermann 1998) and statistics (Pardo 2005).

By definition, $T \geq 0$, with 0 meaning that the distribution is identical to the uniform distribution

53

54

55
56 57 958 
(the observed entropy is equal to the maximum) and higher values signify increased deviation from the uniform case and thus increased inequality. It is important to note that the index is invariant under state switching in the set. For example two individuals, one using the bus $90 \%$ and the remaining modes $10 \%$ of the time, and a second individual using the rail $90 \%$ and the remaining modes $10 \%$ of the time, will be assigned the same Theil value. This doesn't take into consideration which transportation mode is more favourable under a given circumstance. From this perspective, arguments related to equity are not possible by assessing the output of the index alone, and some qualitative discussion of the results is needed. This is also true for the weighted version of the index, as the weighting scheme needs to be decided to reflect equity considerations. Moreover, the uniform level of equality specified by maximum entropy represents a theoretical case that links to egalitarian approaches under the idea of equality of opportunity. However, it is legitimate to expect a certain level of inequality to exist, provided that it is caused by an individual's own choices and not unfavourable circumstances such as having low income (Pereira et al. 2017).

For the purposes of identifying individuals that experience a relative disadvantage, the posterior distributions are compared and contrasted using the Theil index against the state of complete equality characterised by maximum entropy. Since the Oyster card dataset doesn't provide any information related to an individual's preferences or desires, Theil values will be assessed under the assumption that any significant deviations of the individual Theil values from the group population mean could be attributed to particularities of the group (eg. low income, age), treating individual preferences as random fluctuations in the Theil values within the group.

\section{Results}

In this section the posterior distributions for activity types and transportation modes are presented for the three population groups: individuals $>60$ years old, low income individuals $(<£ 15000$ yearly income) and an unconstrained population group. The overarching goal is to emphasize the different accessibility patterns that indicate trans- 1030 port related social exclusion faced by individuals 1031 belonging to different population groups, compared 1032 with the unconstrained population group. For this 1033 task, a popular equality index is used (Theil in- 1034 dex) to quantify equality levels between the three 1035 population groups (Van Wee \& Geurs 2011, Dela- ${ }_{1036}$ fontaine et al. 2011, López et al. 2008). 1037

\subsection{Distributions of activity types ${ }_{1038}$}

The posterior distribution of activity types cor- 1039 responds to the latent $d$ node, expressing the pos- 1040 terior distributions of activity types given the in- 1041 dividual's sociodemographic characteristics, dura- 1042 tion of stay and number of reachable POIs from 1043 the alighting point. Appendix A shows the pos- 1044 terior quantities of $P(d)$ for the trajectories of all 1045 users in the target groups.

1046

\subsubsection{Posterior results}

1047

The posterior quantities for the individuals in 1048 the low income group and $>60$ years old group 1049 were similar to the unconstrained group for all cat- 1050 egories (Figures 13 12 11). For the low income 1051 group one notable difference is the shorter tails 1052 of the daily distributions for the majority of the 1053 individuals for the Employment activity (Figure 1054 12e). Empirically, this could signify reduced flex- 1055 ibility in using public transport to reach this ac- 1056 tivity compared to the unconstrained population ${ }_{1057}$ group. Moreover, the probabilities of Eating and 1058 Drinking (Figure 12a) and Retail (Figure 12c) ac- 1059 tivity types is significantly lower throughout the 1060 day, remaining below the threshold for random 1061 probability allocation for the specified number of 1062 activity types $(<0.2)$. Contrary to the rest of the 1063 population groups, for the $>60$ years old group 1064 the Education and Health (Figure 13b ) category 1065 is characterised by a gradual increase over the later 1066 hours of the day for the majority of the individuals. 1067 This could be attributed to health related activities 1068 as opposed to Education related activities. Again, 1069 the Employment (Figure 13e) activity type seems 1070 to dominate the daily trajectory of this group for 1071 the early hours of the day. This is not surprising 1072 considering the fact that the majority of the indi- 1073 viduals in this group were below the UK national 1074 pension age (63 years for women and 65 years for 1075 


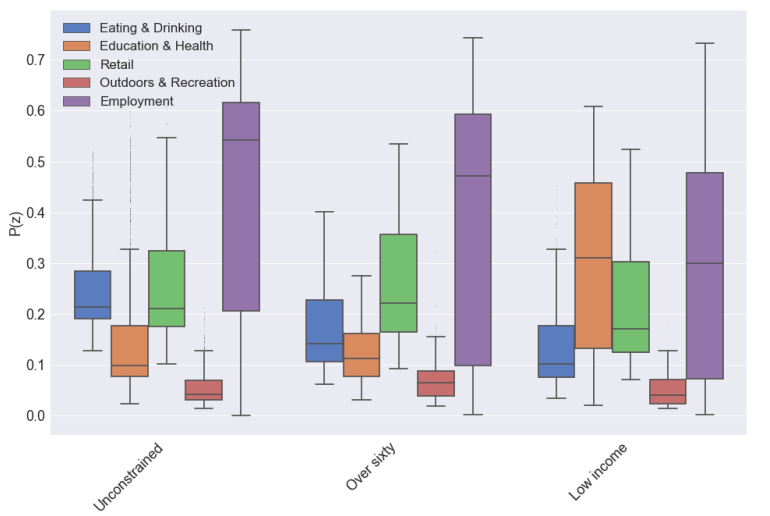

(a) Aggregated activity distribution boxplots for all individuals (weekdays)

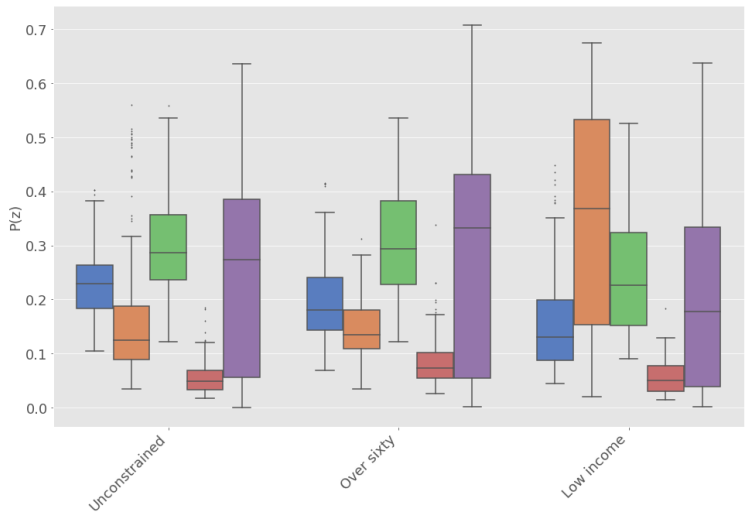

(b) Aggregated activity distribution boxplots for all individuals (weekends)

Figure 5: Aggregated activity type boxplots for the three population groups.

men). Nevertheless, a general shift of this activins with the exception of employment activity which is considerably lower in the weekends.

\subsubsection{Assessing equality levels}

Looking at the distribution of Theil values $(T-$ values) for the three population groups (Figure 6), the low income group has the largest mean compared to the rest of the groups, signalling overall increased inequality levels (at the .05 significance level, one way ANOVA, see Table 4). The equality 1093 assumption made here is that, throughout an indi- 1094 vidual's trajectory, all defined activity types should 1095 be equally reachable by an individual regardless of 1096 factors such as age, income etc., and thus the dis- 1097 tribution of these activity types should approach 1098 the uniform distribution $(T=0)$. Increasing de- 1099 viation from this can be considered deviation from 1100 equality.

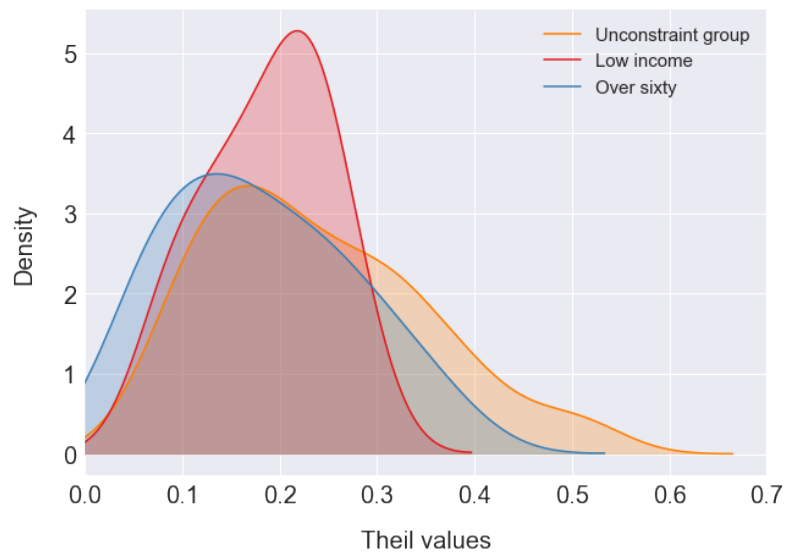

Figure 6: Density plots of Theil indices for the three population groups

Table 4: Desciptive statistics and one way ANOVA for the Theil indices

\begin{tabular}{l|l|l|l|l} 
group & count & mean & std. & $\begin{array}{l}\mathbf{7 5} \\
\text { perc. }\end{array}$ \\
\hline \hline $\begin{array}{l}\text { Uncon- } \\
\text { strained }\end{array}$ & 181 & 0.18 & 0.11 & 0.31 \\
\hline $\begin{array}{l}\text { Over } \\
\text { sixty }\end{array}$ & 30 & 0.12 & 0.09 & 0.25 \\
\hline $\begin{array}{l}\text { Low in- } \\
\text { come }\end{array}$ & 13 & 0.21 & 0.06 & 0.23 \\
\hline \hline
\end{tabular}

(a) Descriptive statistics

\begin{tabular}{l|l|l|l|l} 
& $\begin{array}{l}\text { sum. } \\
\text { sq. }\end{array}$ & df & F & $\begin{array}{l}\text { p- } \\
\text { value }\end{array}$ \\
\hline \hline group & 0.105 & 2.0 & 4.42 & 0.013 \\
\hline Residual & 2.62 & 221.0 & NA & NA \\
\hline \hline
\end{tabular}

(b) One way ANOVA

The distribution of Theil indices for the $>601102$ and unconstrained population groups are similar, 1103 
however, the tail of the unconstrained population group is considerably longer compared to both remaining groups. Under closer examination, these outliers $(>75 \%$ percentile, $T>0.5)$ are characterised by high Employment probabilities at the expense of the rest of activity types. The demographic status of these individuals is composed of a mix of ethnicities, while the place of residence is outer London in most cases. All of the individuals in this sample are full time permanent employed in central London, with activity patterns being limited to $<5$ unique locations.

The outliers $(>75 \%$ percentile $T>0.23$ ) of the low income group on the other hand, are characterised by individuals that are part-time workers and students, again residing in outer London. Their ethnic background is a mix of Asian/Arab/Black or Black British - African and Black or Black British - Caribbean with age spanning from 21 to 38 years old. The household characteristics are lone parents or couples with children. Compared to the outliers of the unconstrained group, the number of unique locations visited is greater. However, the mean distance between these locations $(9.5 \mathrm{~km})$ is much smaller compared to the unconstrained group $(20.4 \mathrm{~km})$. This pattern could be explained by the relatively high rates of travelling by bus and provides evidence of a reduced space where activities can take place compared to the unconstrained group. In the absence of access to individual preference mechanisms, it is difficult to make assertions as to whether this pattern is due to genuine individual choices or whether is related to higher risk of social exclusion. However, given that in London the price of a single bus journey is nearly half the price of rail and taking into consideration the sociodemographic profile of these individuals, it is likely that the observed pattern is due to necessity.

Finally, looking at the demographic characteristics of the outliers of the $>60$ population group $(>75 \%$ percentile, $T>0.24)$, the ethnic backgrounds are mainly White - English/Welsh/Scottish/Northern Irish/Other White residing in outer Greater London with place of employment in Greater London area, inside the M25 motorway. All of the individuals in this percentile were employed full time with annual income ranging between $£ 25,000-100,000$. Similarly to the 1152 unconstrained population group, these individuals 1153 have high Employment activity type probabilities 1154 $(\sim 0.5)$. However, the probabilities for the rest 1155 of the activity types appear to be more balanced. 1156 This population subgroup has the greatest number 1157 of unique visited locations compared to the uncon- 1158 strained and low income groups. However, con- 1159 trary to the low income group, the mean distance 1160 between these locations is slightly larger $(10 \mathrm{~km})$, a 1161 fact which could be explained by the higher rate of 1162 travelling by rail for activity Eating and Drinking. 1163

\subsection{Distribution of transportation 1164 modes

The next posterior quantity of interest is the dis- 1166 tribution of transportation modes for each individ- 1167 ual in the population groups. This corresponds to 1168 the latent $m$ node of the model 4 and relates to the 1169 mobility element of the Capabilities set. Similarly 1170 to the $d$ node, the results for the unconstrained, low 1171 income and $>60$ population groups are presented 1172 in Appendix B. The segmentation per activity type 1173 was made by taking the one with the highest prob- 1174 ability from each individual activity distribution at 1175 visited location.

\subsubsection{Posterior quantities}

1177

Compared to the unconstrained population 1178 group, for the low income group the probabili- 1179 ties of using the rail to reach activity type Eat- 1180 ing and Drinking are significantly lower, with bus 1181 being the predominant transport mode for this ac- 1182 tivity type (Figures 15a, 15b 15c). The posterior 1183 probabilities for the Education and Health (Figure 1184 15d 15e 15f) activity type are slightly higher for 1185 using the bus compared to rail services, and the 1186 same holds for the Retail (Figure 15g 15h 15i) ac- 1187 tivity type. In terms of the overall shape of the ${ }_{1188}$ distributions, Retail seems to follow the trend ob- 1189 served with the unconstrained population group, 1190 coinciding with retail shops' most popular shop- 1191 ping times. Looking at the Employment (Figure 1192 $15 \mathrm{~m} 15 \mathrm{n} 15 \mathrm{o}$ activity type, the shape of poste- 1193 rior distributions for bus, rail and tram services 1194 is significantly wider throughout the day, com- 1195 pared to the unconstrained sample, characterised 1196 by two peaks, in the morning and early afternoon. ${ }_{1197}$ 
This pattern most likely reflects the varying schedule of part-time workers. Finally, for this population group, only one individual was attributed with reaching Outdoors and Recreation (Figure 15j, 15k 15l activity type with higher probability of using the rail services.

For the $>60$ population group, the probabilities of using the bus and using rail to reach activity type Eating and Drinking seem to complement each other, with the probabilities of bus use being higher in the morning/afternoon and rail being higher in the afternoon/evening hours (Figure 16a. 16b). Overall, using the bus versus rail is similar for Education and Health, with rail services appearing to have a slightly shifted distribution mode toward the afternoon hours (Figure 16d, 16e). Using the different transportation modes to reach Retail (Figure 16g, 16h, 16i) appears to be similar with the rest of population groups. However, the distribution of transport modes used throughout the day appears to be wider for a significant number of individuals. Moreover, compared to the rest of focus groups, more people are found to be using the bus to reach Outdoors and Recreation activities.

Figures 7 and 8 below show aggregated boxplots of the transportation modes posterior distributions for all in individuals in the three population groups, categorised by weekdays and weekends. As it can be seen, the overall use of public transport for activity Employment is generally lower in the weekends for the unconstrained and $>60$ group, particularly for using the bus. For the low income group, using rail for reaching activity Eating and Drinking is lower in the weekends compared to weekdays. On the other hand, weekdays dominate the use of public transport to reach Education and Health for the $>60$ population group.

\subsubsection{Assessing equality levels}

The equality assumption made here is similar to potential accessibility: all transportation modes should be equally available regardless of any personal or place based characteristics. It is important to note that this assumption is useful only in the context of benchmarking the individual Theil values, as it is well known that the public transportation network is designed so that each mode complements the other. Moreover, as in the case
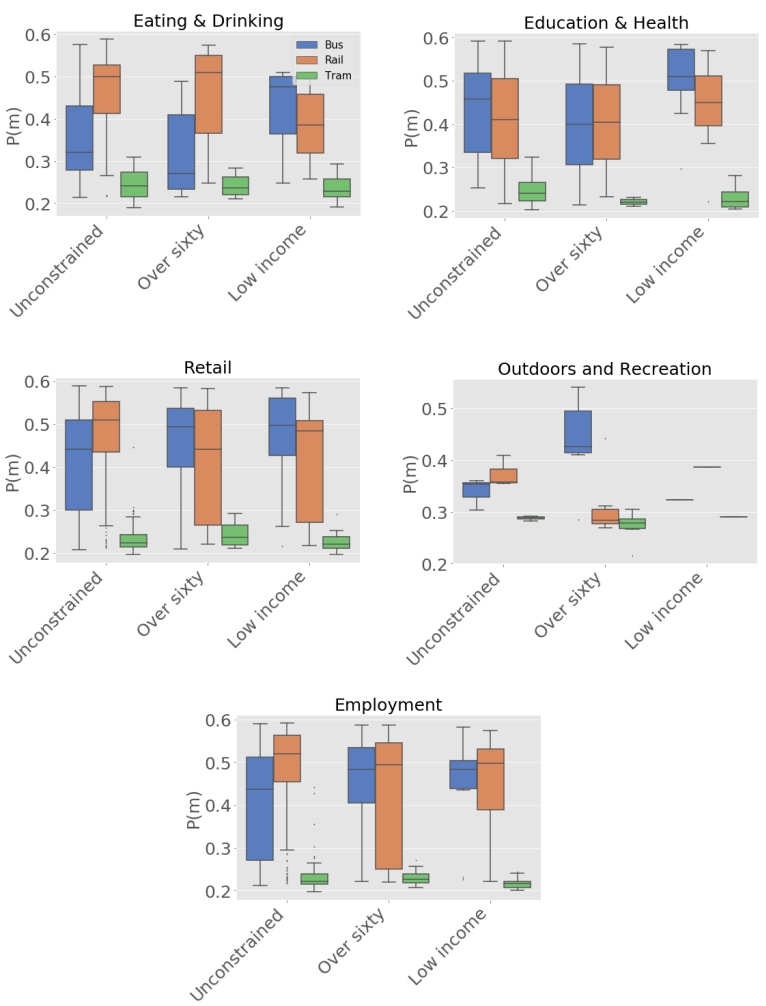

Figure 7: Aggregated transportation mode boxplots (weekdays)

of Tram services in London, some transportation 1245 modes are operate on a local scale only, so by de- 1246 fault are not readily available to the general pop- 1247 ulation. Nevertheless, by evaluating the individual 1248 Theil indices in a relative way, it is possible to iden- 1249 tify cases where the use of a transport mode is not 1250 possible due to factors beyond the control of an in- 1251 dividual (such as their sociodemographic status), a 1252 fact which could relate to transport disadvantage. 1253

Figure 9 below shows density plots of Theil in- 1254 dices for the posterior transportation mode distri- 1255 butions for each population group: 1256

Similar to Section 3.3.1, a one way ANOVA test ${ }_{1257}$ was performed which resulted in failure to reject 1258 the null hypothesis, concluding that the distribu- 1259 tions belong to the same population (Table 5). 1260 However, this result could be an artifact of the 1261 lower cardinality of the transportation mode set, 1262 particularly considering the very low use of Tram 1263 services, resulting in small differences in Theil val- 1264 ues. 

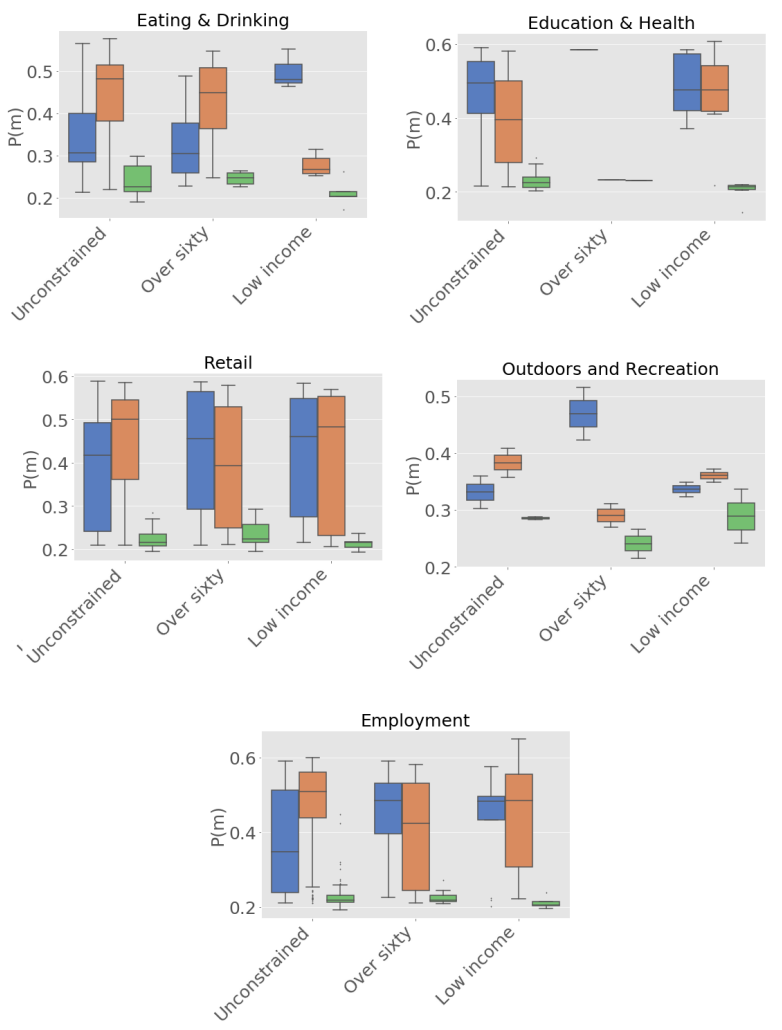

Figure 8: Aggregated transportation mode boxplots (weekends)

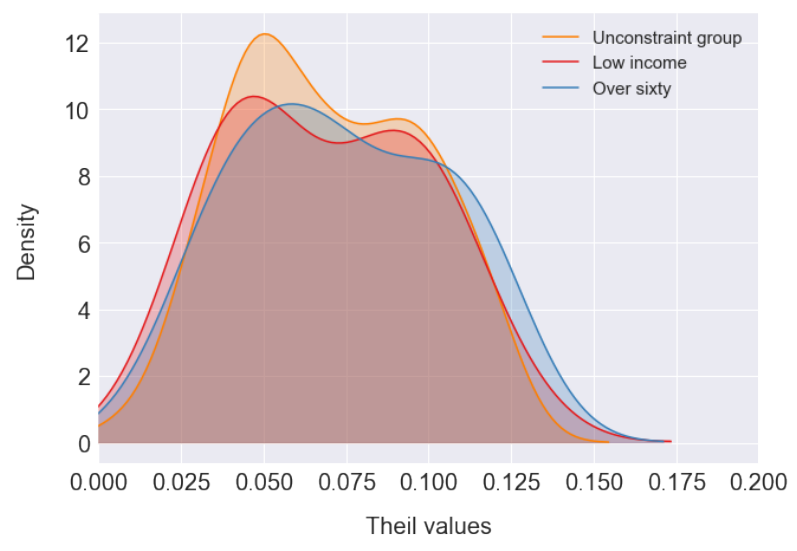

Figure 9: Density plots of mobility Theil indices for the three population groups
Exploring the Theil values distributions qualitatively, one notices a bimodality in all three population groups, meaning that, for those individuals, the use of one transportation mode dominates over
Table 5: Descriptive statistics and one way ANOVA for the Theil indices of transportation modes

\begin{tabular}{l|l|l|l|l} 
group & count & mean & std. & $\begin{array}{l}\mathbf{7 5} \\
\text { perc. }\end{array}$ \\
\hline \hline $\begin{array}{l}\text { Uncon- } \\
\text { strained }\end{array}$ & 181 & 0.07 & 0.03 & 0.1 \\
\hline $\begin{array}{l}\text { Over } \\
\text { sixty }\end{array}$ & 30 & 0.073 & 0.029 & 0.11 \\
\hline $\begin{array}{l}\text { Low in- } \\
\text { come }\end{array}$ & 13 & 0.06 & 0.02 & 0.09 \\
\hline \hline
\end{tabular}

(a) Descriptive statistics

\begin{tabular}{l|l|l|l|l} 
& $\begin{array}{l}\text { sum. } \\
\text { sq. }\end{array}$ & df & F & $\begin{array}{l}\text { p- } \\
\text { value }\end{array}$ \\
\hline \hline group & $0.0003 \S$ & 2.0 & 0.23 & 0.79 \\
\hline Residual & 0.18 & 221.0 & NA & NA \\
\hline \hline
\end{tabular}

(b) One way ANOVA

all others. It is interesting to observe that, in con- 1270 trast to the unconstrained population group, the 1271 second mode of the low income group is attributed 1272 to very high probabilities of Bus use. Examining 1273 the outliers $(>75 \%$ percentile, $T>0.1$ ) of the low 1274 income distribution, one notices that the majority 1275 of individuals in this set are a subset of the low 1276 income outliers of Section 3.3.1. This fact provides 1277 further evidence of the potential for social exclu- 1278 sion for these individuals.

\subsection{Activity and mobility dynamics 1280}

In this section, the posterior results of tran- 1281 sition matrices $T_{m}$ and $T_{z}$ are presented. Intu- 1282 itively, these matrices capture the transition dy- 1283 namics for the accessibility and mobility modules 1284 of model shown in Figure 4 taking into consider- 1285 ation the effects of external factors as individuals 1286 transition from one transportation mode/activity ${ }_{1287}$ to another during the trajectory. It is important to 1288 note that, contrary to $T_{m}$ where the transportation 1289 mode states are inferred using the observed Oyster 1290 card modes, $T_{z}$ captures the transition dynamics of 1291 inferred activity types. The results for the uncon- 1292 strained, low income and $>60$ population groups 1293 are presented per activity type in appendix C. $\quad 1294$ 


\subsubsection{Activity type transitions posterior results}

Figure 17 in appendix C presents the posterior distributions for each element of the activity type transition matrix $T_{z}$ for all individuals in the unconstrained population sample. As it can be seen, the transition patterns from activity type Employment to all other activities vary significantly between individuals, ranging from $0.2<P\left(T_{z}\right)<0.8$, with an overall mean probability of $\approx 0.4$ for the transition from all other types to Employment making this the dominant sequence in this group. Looking at the transition between Education and Health and Education and Health, individuals seem to be divided into two clusters, one with relatively low probability $P\left(T_{11}\right)<0.2$ and one with probabilities $P\left(T_{11}\right)>0.2$, a behaviour which could be attributed to the students/pupils in the sample. Relatively high probabilities for many individuals are also observed between transitions Retail/Retail, Eating and Drinking/Retail.

Results for the low income population group are shown in Figure 18. The transition patterns are very similar with the unconstrained population group, however in this case, the probabilities of transitioning from Employment to all other activity types is lower on sample population level $P\left(T_{z}\right)<0.2$.

Finally, Figure 19 presents the results for the over sixty years old population group. Again, the results here are very similar to the rest of the target groups, with the dominant transition sequences being between Employment and the rest of activity types.

\subsubsection{Transportation mode transitions posterior results}

Looking at the unconstrained population group (Figure 20), there is a clear tendency to persistently transition from rail services to rail services $\left(T_{m_{11}}\right)$ with a population level probability of $P\left(T_{m_{11}}\right) \approx 0.65$, a behaviour which could largely be attributed to commuting to employment activities. The transition probabilities from bus to rail are also relatively high in the sample $\left(P\left(T_{m_{01}}\right) \approx 0.45\right)$, in par with transition from bus to bus $\left(P\left(T_{m_{00}}\right) \approx\right.$ $0.475)$. The transition from rail to bus on the other hand $\left(P\left(T_{m_{10}}\right) \approx 0.305\right)$ is relatively low compar- atively, indicating that, on average, individuals of 1342 this sample seem not to prefer finishing their jour- 1343 ney on the bus if rail was the prior choice. As 1344 expected, due to the lack of tram transactions in 1345 the sample but also due to the limited coverage of ${ }_{1346}$ tram services, on average the transition probabili- 1347 ties between the rest of transportation modes and 1348 tram is relatively small. This is confirmed by the 1349 uniform allocation of probabilities between tram 1350 and the rest of modes. This pattern is similar to 1351 the over sixty and low income population groups. 1352

Transition probability patterns for the over sixty 1353 population group (Figure 21) are different, provid- 1354 ing evidence that, on average, there is increased 1355 likelihood of using the bus persistently throughout 1356 a trajectory $\left(P\left(T m_{00}\right) \approx 0.73\right)$. The inverse is true ${ }_{1357}$ for transitioning from bus to rail $\left(P\left(T m_{01}\right) \approx 0.24{ }_{1358}\right.$ ). Transitioning from rail to all other modes appear 1359 to be less clustered $\left(P\left(T m_{11}\right) \approx 0.40, P\left(T m_{10}\right) \approx 1360\right.$ 0.45 ), indicating perhaps the less frequent use of ${ }_{1361}$ rail services in this target group. 1362

Finally, the low income population group (Figure 1363 22 provides evidence of a broader transition prob- 1364 abilities spread amongst individuals for the bus 1365 services, with a tendency to prefer using the bus 1366 throughout the trajectory $\left(P\left(T m_{00}\right) \approx 0.55\right)$ com- 1367 pared to transitioning from bus to rail $\left(P\left(T m_{01}\right) \approx{ }_{1368}\right.$ 0.41). The overall pattern of rail use is similar to 1369 the over sixty population group, showing a uni- 1370 form distribution of transitions between rail/bus 1371 and rail $/$ rail $\left(P\left(T m_{10}\right) \approx 0.46, P\left(T m_{11}\right) \approx 0.43\right) . \quad 1372$

\subsubsection{Assessing equality levels}

This element of the capabilities set is aiming to 1374 quantify the dynamic component between different 1375 activity types/transportation modes through the 1376 use of external factor informed transition matrices. 1377 Regarding activity types, the underlying assump- 1378 tion being made is that an individual is less likely $\quad 1379$ to be socially excluded if they maintain a uniform 1380 level of interaction with the available activities, as 1381 this translates to more frequent trips per activity 1382 type which is thought to map to increased levels of ${ }_{1383}$ social involvement (Schönfelder \& Axhausen 2003). ${ }_{1384}$ A similar rationale holds for the interaction with 1385 public transport modes as expressed through the 1386 mobility transition matrix, in that increased lev- 1387 els of transition between modes could translate to 1388 

groups.

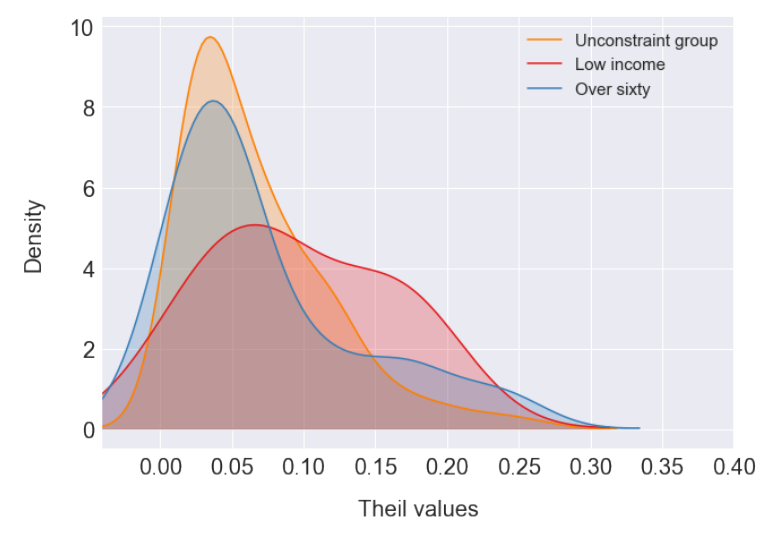

(a) Density plots of Theil indices for the activity types transition matrix

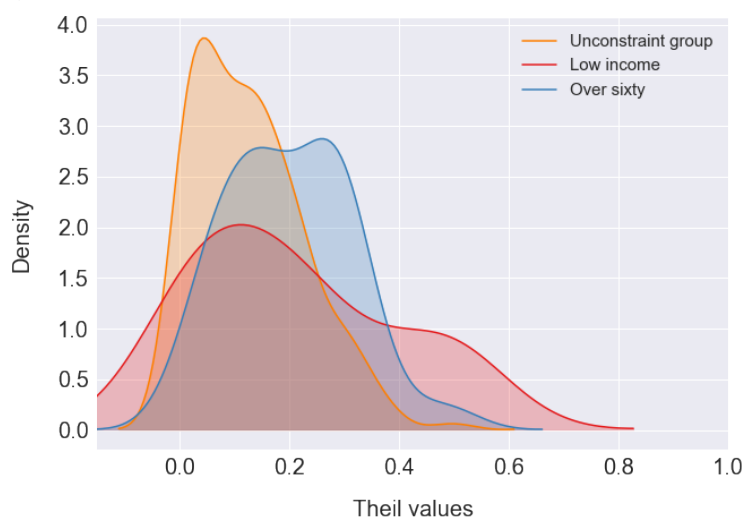

(b) Density plots of Theil indices for the mobility transition matrix

Figure 10: Density plots of Theil indices for the activity types transition matrix.

an expansion of the set of activities within reach. Figures $10 \mathrm{a}$ and $10 \mathrm{~b}$ below show the distribution of Theil values for $T_{z}, T_{m}$ for the three population

The ANOVA test for the three population groups (Table 6) failed to reject the null hypothesis (same distributions), however for the low income group there are some outliers that seem to have increased Theil values.

Similarly to 3.3.1, the employment status of outliers $(>75 \%$ percentile, $T>0.2$ ) of the low income group are a mixture of student, part-time and full time workers, residing in outer Greater London. Not surprisingly, these individuals are characterised by increased probabilities of transitions related to Health and Education (for the student and part-time employed individual) and increased
Table 6: Descriptive statistics and one way ANOVA for the Theil indices of activity transition matrices

\begin{tabular}{l|l|l|l|l} 
group & count & mean & std. & $\begin{array}{l}\mathbf{7 5} \\
\text { perc. }\end{array}$ \\
\hline \hline $\begin{array}{l}\text { Uncon- } \\
\text { strained }\end{array}$ & 181 & 0.06 & 0.05 & 0.094 \\
\hline $\begin{array}{l}\text { Over } \\
\text { sixty }\end{array}$ & 30 & 0.072 & 0.066 & 0.10 \\
\hline $\begin{array}{l}\text { Low in- } \\
\text { come }\end{array}$ & 13 & 0.097 & 0.06 & 0.15 \\
\hline \hline
\end{tabular}

(a) Descriptive statistics

\begin{tabular}{l|l|l|l|l} 
& $\begin{array}{l}\text { sum. } \\
\text { sq. }\end{array}$ & df & F & $\begin{array}{l}\text { p- } \\
\text { value }\end{array}$ \\
\hline \hline group & 0.007 & 2.0 & 1.21 & 0.298 \\
\hline Residual & 0.55 & 221.0 & NA & NA \\
\hline
\end{tabular}

(b) One way ANOVA

transition probabilities related to Employment for 1406 the full time workers. The latter is the same for ${ }_{1407}$ nearly all outliers of the unconstrained and over ${ }_{1408}$ sixty population group. 1409

The ANOVA test for the distribution of individ- 1410 ual transportation mode transition (Table 7) ma- 1411 trices has rejected the null hypothesis $(F-$ value $=1412$ $8.908, p=0.0002)$ stating that the Theil distribu- 1413 tions are different. Looking at the mean Theil val- 1414 ues for all three population groups in Figure 10b, 1415 the over sixty and low income groups seem to have 1416 similar inequality levels $(\bar{T}=0.21$ for low income, 1417 $\bar{T}=0.20$ for over sixty). However, for the indi- 1418 viduals with Theil values belonging to the tails of 1419 the distribution, the levels of inequality seem to be 1420 particularly high. The qualitative profile of those ${ }_{1421}$ individuals is similar to the ones of activity tran- 1422 sition matrices (mixture of employment statuses 1423 and residing in Outer London) with high transi- 1424 tion probabilities of using a particular mode (Bus 1425 or Rail).

1426

\section{Discussion}

1427

The link between social exclusion and transport ${ }_{1428}$ disadvantage is a complex one that has been ap- 1429 proached in different ways in the literature. Hav- 1430 ing as a starting point the Capabilities Approach, 1431 
Table 7: Descriptive statistics and one way ANOVA for the Theil indices of transportation mode transition matrices

\begin{tabular}{l|l|l|l|l} 
group & count & mean & std. & $\begin{array}{l}\mathbf{7 5} \\
\text { perc. }\end{array}$ \\
\hline \hline $\begin{array}{l}\text { Uncon- } \\
\text { strained }\end{array}$ & 181 & 0.12 & 0.09 & 0.19 \\
\hline $\begin{array}{l}\text { Over } \\
\text { sixty }\end{array}$ & 30 & 0.20 & 0.10 & 0.27 \\
\hline $\begin{array}{l}\text { Low in- } \\
\text { come }\end{array}$ & 13 & 0.21 & 0.17 & 0.29 \\
\hline \hline
\end{tabular}

(a) Descriptive statistics

\begin{tabular}{l|l|l|l|l} 
& $\begin{array}{l}\text { sum. } \\
\text { sq. }\end{array}$ & df & F & $\begin{array}{l}\text { p- } \\
\text { value }\end{array}$ \\
\hline \hline group & 0.17 & 2.0 & 8.908 & 0.0002 \\
\hline Residual & 2.22 & 221.0 & NA & NA \\
\hline
\end{tabular}

(b) One way ANOVA

this study proposed an accessibility framework to quantify the links between limited access to opportunities and reduced access to public transport using unlabelled mobility data.

The general trend for distribution of activity types throughout the day was found to be similar in the three focus population groups, a fact which is not surprising given that activity types were imputed, and not observed, from secondary data. However, including assumptions about the nature of the sociodemographic background of individuals allowed shaping of the head and tails of these distributions, particularly for the Employment activity type.

The low income population group was found to have smaller probabilities of activity type Eating and Drinking, which could be linked to the reduction of the capability to use public transport to reaching entertainment related activities. Although it is hard to make assertions due to the small sample size, it is nevertheless interesting to observe that the activity type that has the lowest probability range is the one that is the most elastic, compared to Employment or Education and Health for example. On the other hand, the probabilities of activity type Education and Health are higher for the low income group. Considering that a large number of individuals in this population group are 1459 students, this fact comes as no surprise.

1460

For all population groups, activity type Outdoors 1461 and Recreation was found to have the lowest prob- 1462 ability compared to the rest of the activity types. ${ }_{1463}$ Besides the limited number of POIs in this category ${ }_{1464}$ type, the prior assumptions of participating in this 1465 activity type were weak relative to the rest of activ- 1466 ity types (the mean value was $\approx 0.13$ for all individ- ${ }_{1467}$ uals in the Oyster sample). This finding, together ${ }_{1468}$ with the fact that Outdoors and Recreation had the ${ }_{1469}$ highest accuracy of prediction (following Employ- 1470 ment) makes the assertion of absence of such ac- 1471 tivity types from the dataset plausible, as opposed 1472 to being an artefact of the modelling process. $\quad{ }_{1473}$

A further breakdown of results can be made 1474 by examining the distributions of transportation 1475 mode use in relation to each activity type. For 1476 the unconstrained population group, this reveals 1477 increased diversity in the probability distributions 1478 among individuals on the relative use of public 1479 transport, particularly for Eating and Drinking 1480 and Education and Health activity types. This 1481 could be an indication of the varying capability lev- 1482 els experienced by different people when reaching 1483 these activities. Overall, for this population group, 1484 the probability of using Rail services to reach the ${ }_{1485}$ different activity types is higher compared to the 1486 rest of the modes. The exception is Education and ${ }_{1487}$ Health where Bus seems to be considerably higher ${ }_{1488}$ $(\approx 0.42$ for Bus and $\approx 0.36$ for Rail). Assuming 1489 that these activities predominantly map to individ- 1490 uals that are either students or people that reach 1491 for medical care, decreased levels of Rail use com- 1492 pared to Bus provides evidence of reduced capabil- 1493 ity of using the Rail services by those individuals. 1494

The use of Bus services is also the predominant 1495 mode of transport for the low income group for all 1496 activity types, a finding that is in line with existing 1497 evidence (Transport for London 2011). This should 1498 hardly come as a surprise, as cost is a significant 1499 barrier to transport in London, particularly for rail 1500 services. Other reasons mentioned in the literature 1501 for increased bus use are the possession of bus/rail 1502 cards for the low income groups. However, none 1503 of the individuals in the group reported possessing 1504 one in the LTDS. The predominant use of bus for 1505 people in this group could also be the main rea- 1506 
son for the observed geographical pattern, which is characterised by a tendency to avoid inner London. This fact, combined with reduced participation in activities as determined by the increased Theil index for this group, provides evidence of transport disadvantage compared to the rest of the groups.

Furthermore, the distribution of transportation mode use for the Employment activity type has been found to have a distinct multimodal shape throughout the day, characterised by relatively high probabilities in the morning and afternoon. This pattern could be explained by the mixture of employment statuses of the individuals in this group: full-time employed, part-time employed and students.

Compared to the rest of the groups, individuals in the $>60$ sample have wider distributions of public transport use throughout the day, spanning a temporal window between morning and early evening. It is difficult to interpret this shape, as from a data driven perspective, this group had the least number of transactions on average $(\approx 30$ transactions per individual) compared to the rest of the groups $(\approx 32$ for low income and $\approx 38$ for the unconstrained group) which contributes to increased uncertainty of estimates. This fact coincides with evidence of non-travel (people who do not make trips) for this population group (Transport for London 2011) in London. From this perspective, it is difficult to make assertions of increased capability of using the public transport network throughout the day compared to the unconstrained population group.

The general pattern of transition probabilities between activity types was found similar in the three population groups. One notable exception was the relatively low transition probabilities of Employment for the low income group. Empirically, this pattern could be attributed to the nature of working status of the individuals in the sample, half of which were students, $25 \%$ full-time and $25 \%$ part-time employed. Moreover, this population group was found to have a larger distribution mean, providing evidence of less uniform transitions between activities. Indeed examining the outliers of the Theil distribution, one notices increased transition probabilities for either education or employment related activities, depending on the individual's employment status.

Looking at the results of the mobility transi- 1556 tion matrix, a number of interesting transporta- 1557 tion habits are revealed. For a significant num- 1558 ber of individuals belonging in the unconstrained 1559 population group, the use of Rail services seem to 1560 be persisting throughout their trajectory, charac- 1561 terised by high Rail/Rail and low Rail/Bus tran- 1562 sition probabilities. On the other hand, the in- 1563 verse seems to be true for the over sixty and low 1564 income population groups, with high Bus/Bus and 1565 low Bus/Rail probabilities. This transition pattern 1566 is less uniform for the low income group, judging 1567 from the increased Theil values. Although it is dif- 1568 ficult to make assumptions on the drivers behind 1569 this modal split pattern, it seems that, besides fac- 1570 tors commonly mentioned in the literature such as 1571 egress and waiting time (?), sociodemographic fac- 1572 tors (such as employment status and income) also 1573 play a role in the modal split habits of individuals. 1574 In each case, the lack of sensitivity in switching 1575 between different public transport modes could be 1576 regarded as a reduced capability of using the public 1577 transportation network that can result to transport 1578 disadvantage.

1579

In terms of individual levels of equality as de- 1580 termined using the Theil index, the distribution of 1581 individual Theil values for the low income group 1582 is characterised by a statistically significant larger 1583 mean compared to the rest of the population 1584 groups. This provides evidence of increased lev- 1585 els of inequality experienced by this group, as the 1586 range of activities that are being reached is nar- 1587 rower.

Examining the sociodemographic variables of 1589 outliers of this distribution (individuals belonging 1590 over the $75 \%$ of the Thiel distribution, a total of 1591 10 individual) using the matched Oyster card and 1592 LTDS IDs, further probing of the personal char- 1593 acteristics contributing to exclusion from activi- 1594 ties and access to transportation modes can be de- 1595 duced: $90 \%$ belong to black, Asian and minority 1596 ethnic backgrounds, $70 \%$ are women, all of them 1597 report income earned below $£ 15,000$ and all of ${ }_{1598}$ them reside in outer Greater London. Moreover, 1599 the labour profile for these individuals is more un- 1600 stable, with 8/10 people being either part-time em- 1601 ployed or students. 
In contrast, the sociodemographic profile of the Thiel outliers of the unconstrained population group (16 individuals) consisted of $44 \%$ belonging to to black, Asian and minority ethnic backgrounds, $62 \%$ women, all of them earning $£ 25,000$ or more and $67 \%$ residing in outer Greater London areas with 15/16 being full time employed. The sociodemographic profile of the $>60$ population group is similar to the unconstrained group (11 individuals) with $63 \%$ women, all of them earning $£ 25,000$ or more and $80 \%$ residing in outer Greater London areas and 9/11 full time employed. The ethnic background however of the outliers in this group is different with $10 \%$ belonging to black, Asian and minority ethnic backgrounds and the rest are White/British white. Judging from the above profiles, it is clear that the low income group is characterised by most of the risk factors that could result in social exclusion.

\section{Conclusions}

This study proposed a novel approach to evaluating individual accessibility by framing the modelling methodology through the CA. Following the main concepts of the CA and the way they are related, the different components that shape an individual's ability to reach opportunities are explicitly modelled in a probabilistic way through the notions of latent capabilities and observed functionings. The potential of the proposed methodological framework to evaluate individual based transport based social exclusion was assessed through a case study using London's transport data. It was found that the proposed framework could identify individuals that exhibit high risk of social exclusion by comparing the distributions of the capabilities sets. Limitations of this approach can be summarised to the nature of passively generated mobility data. Since such data were generated by the service provider for reasons other than the one used in this study, any generalisations to population level characteristics should be made keeping this important consideration in mind. For example, population groups that are thought to present high risk of transport related social exclusion such as the unemployed, disabled and retired were not represented in the sample. It would be of great value if the analysis was repeated with these groups, as it would demonstrate the degree of robustness of the 1650 proposed methodological framework. 1651

Related to the above, it is important to note that 1652 while this study represented an individual's poten- 1653 tial accessibility to activities and potential mobil- 1654 ity using public transport through the Bayesian 1655 network structure, access to an individual's actual 1656 "wants" and "desires" behind their choices remains ${ }_{1657}$ out of reach and can only be uncovered through 1658 extensive qualitative studies. For example, people 1659 with lower income may choose to eat and drink out 1660 less due to lack of resources. Transport accessibil- ${ }_{1661}$ ity may be a factor, but its effect might be exag- 1662 gerated by the lack of access to the drivers behind 1663 the choices made by those individuals. Neverthe- ${ }_{1664}$ less, the current structure of the proposed model 1665 could be used to identify deviations from the aver- 1666 age equality levels so that further investigation can $\quad{ }_{1667}$ be undertaken. Future direction will be steered to- 1668 wards a qualitative validation of the findings. $\quad 1669$

Furthermore, the varying sample size of Oyster 1670 card data for the different population groups has an 1671 impact on the geographic representativeness of the 1672 study area. While for the unconstrained and > 601673 sample the visited locations appear to be uniform 1674 throughout the study area, the visited locations 1675 of low income group appear to span radially from 1676 outer to inner London (see Figure 3). Although 1677 a positive correlation exists between the index of 1678 deprivation and the visiting locations of the low in- 1679 come group (OLS slope 0.013 compared to a neg- 1680 ative correlation for the unconstrained group with 1681 OLS slope -0.044 and the $>60$ group with OLS 1682 slope -0.001) which intuitively is what would one 1683 expect, it is difficult to make any firm assertions 1684 regarding the geographic representativeness of this 1685 population group.

1686

With regards to the temporal extent of the study, 1687 the available dataset did not allow any deeper eval- 1688 uation on the way individuals adjust their activ- 1689 ity/travel behaviour in the face of an event that 1690 could impact accessibility. Such an event can be re- 1691 lated to personal characteristics (such as a change 1692 in employment status) or can be infrastructure re- 1693 lated (for example, an introduction of a new public ${ }_{1694}$ transport connection. A future direction could in- 1695 volve using an extended time span together with 1696 information on significant events to assess whether 1697 
an adaption of behaviour is represented in the evolution of mobility/accessibility nodes of the model.

In light of the ever increasing trend of urbanisation, accessibility is likely to be a major problem for future cities, as current infrastructure will be stressed to accommodate the needs of an increasing urban population. With the levels of inequality in transport likely to increase as a result of competition for resources, policy makers will need more information on the causes of transport related social exclusion. To that extend, new technologies combined with big data that provide interpretable results could provide evidence to promote equity.

\section{References}

Alkire, S. (2008), 'Using the capability approach: Prospective and evaluative analyses", The Capability Approach: Concepts, Measures and Applications, Cambridge University Press, Cambridge pp. 26-50.

Anda, C., Erath, A. \& Fourie, P. J. (2017), 'Transport modelling in the age of big data', International Journal of Urban Sciences 21(sup1), 1942.

Bagchi, M. \& White, P. R. (2005), 'The potential of public transport smart card data', Transport Policy 12(5), 464-474.

Bantis, T. \& Haworth, J. (2017), 'Who you are is how you travel: A framework for transportation mode detection using individual and environmental characteristics', Transportation Research Part C: Emerging Technologies 80, 286-309.

Bantis, T. \& Haworth, J. (2019), 'Non-employment activity type imputation from points of interest and mobility data at an individual level: How accurate can we get?', ISPRS International Journal of Geo-Information 8(12), 560.

Ben-Akiva, M. (1979), 'Disaggregate travel and mobility choice models and measures of accessibility', Behavioural travel modelling .

Beyazit, E. (2011), 'Evaluating social justice in transport: lessons to be learned from the capability approach', Transport reviews 31(1), 117134.
Burns, L. D. (1980), 'Transportation, temporal, 1741 and spatial components of accessibility'. 1742

Casas, I. (2007), 'Social exclusion and the disabled: 1743 An accessibility approach', The Professional Ge- 1744 ographer 59(4), 463-477.

Chikaraishi, M. (2017), Mobility of the elderly, in 1746 'Life-oriented behavioral research for urban pol- 1747 icy', Springer, pp. 267-291.

1748

Chikaraishi, M., Jana, A., Bardhan, R., Varghese, 1749 V. \& Fujiwara, A. (2017), 'A framework to ana- 1750 lyze capability and travel in formal and informal 1751 urban settings: a case from mumbai', Journal of 1752 transport geography 65, 101-110.

1753

Church, A., Frost, M. \& Sullivan, K. (2000), 1754 'Transport and social exclusion in london', 1755 Transport Policy 7(3), 195-205.

1756

Cohen, J. E. \& Kempermann, J. (1998), Compar- 1757 isons of Stochastic Matrices with Applications in 1758 Information Theory, Statistics, Economics and 1759 Population, Springer Science \& Business Media. 1760

Currie, G., Richardson, T., Smyth, P., Vella- 1761 Brodrick, D., Hine, J., Lucas, K., Stanley, 1762 J., Morris, J., Kinnear, R. \& Stanley, J. 1763 (2010), 'Investigating links between transport 1764 disadvantage, social exclusion and well-being in 1765 melbourne-updated results', Research in trans- 1766 portation economics $\mathbf{2 9}(1)$, 287-295.

1767

Daziano, R. A., Miranda-Moreno, L. \& Heydari, 1768 S. (2013), 'Computational bayesian statistics 1769 in transportation modeling: from road safety 1770 analysis to discrete choice', Transport reviews 1771 33(5), 570-592.

1772

Delafontaine, M., Neutens, T., Schwanen, T. \& 1773 Van de Weghe, N. (2011), 'The impact of open- 1774 ing hours on the equity of individual space-time 1775 accessibility', Computers, environment and ur- 1776 ban systems 35(4), 276-288. 1777

Delbosc, A. \& Currie, G. (2011), 'The spatial con- 1778 text of transport disadvantage, social exclusion 1779 and well-being', Journal of Transport Geography 1780 19(6), 1130-1137. 
Dodson, J., Buchanan, N., Gleeson, B. \& Sipe, N. (2006), 'Investigating the social dimensions of transport disadvantage - i. towards new concepts and methods', Urban Policy and Research 24(4), 433-453.

Evans, G. (2009), 'Accessibility, urban design and the whole journey environment', Built environment 35(3), 366-385.

Farrington, J. \& Farrington, C. (2005), 'Rural accessibility, social inclusion and social justice: towards conceptualisation', Journal of Transport geography 13(1), 1-12.

Farrington, J. H. (2007), 'The new narrative of accessibility: its potential contribution to discourses in (transport) geography', Journal of Transport Geography 15(5), 319-330.

Geurs, K. T. \& Van Wee, B. (2004), 'Accessibility evaluation of land-use and transport strategies: review and research directions', Journal of Transport geography 12(2), 127-140.

Golob, T. F. \& McNally, M. G. (1997), 'A model of activity participation and travel interactions between household heads', Transportation Research Part B: Methodological 31(3), 177-194.

Goodman, A., Jones, A., Roberts, H., Steinbach, R. \& Green, J. (2014), "we can all just get on a bus and go': Rethinking independent mobility in the context of the universal provision of free bus travel to young londoners', Mobilities 9(2), 275293.

Hananel, R. \& Berechman, J. (2016), 'Justice and transportation decision-making: The capabilities approach', Transport Policy 49, 78-85.

Hansen, W. G. (1959), 'How accessibility shapes land use', Journal of the American Institute of planners 25(2), 73-76.

Hickman, R., Cao, M., Mella Lira, B., Fillone, A. \& Bienvenido Biona, J. (2017), 'Understanding capabilities, functionings and travel in high and low income neighbourhoods in manila', Social Inclusion 5(4), 161-174.
Kamruzzaman, M., Yigitcanlar, T., Yang, J. \& ${ }_{1823}$ Mohamed, M. A. (2016), 'Measures of transport- 1824 related social exclusion: A critical review of the 1825 literature', Sustainability 8(7), 696.

1826

Kenyon, S. (2003), Understanding social exclusion 1827 and social inclusion, in 'Proceedings of the In- ${ }_{1828}$ stitution of Civil Engineers-Municipal Engineer', 1829 Vol. 156, Thomas Telford Ltd, pp. 97-104. 1830

Kwan, M.-P. (2013), 'Beyond space (as we knew ${ }_{1831}$ it): Toward temporally integrated geographies 1832 of segregation, health, and accessibility: Space- 1833 time integration in geography and giscience', $A n-1834$ nals of the Association of American Geographers 1835 103(5), 1078-1086.

1836

López, E., Gutiérrez, J. \& Gómez, G. (2008), 1837 'Measuring regional cohesion effects of large- 1838 scale transport infrastructure investments: an 1839 accessibility approach', European Planning Stud- 1840 ies 16(2), 277-301.

184

Lucas, K. (2012), 'Transport and social exclusion: 1842 Where are we now?', Transport policy 20, 105- 1843 113.

Lucas, K. \& Porter, G. (2016), 'Mobilities and 1845 livelihoods in urban development contexts: in- 1846 troduction.', Journal of transport geography. ${ }_{1847}$ 55, 129-131.

1848

Maciel, V., Kuwahara, M., Fronzaglia, M., 1849 Scarano, P. \& Muramatsu, R. (2015), Ac- 1850 cessibility and well-being: Measuring urban 1851 (im)mobility as deprivation, in '2015 Human De- 1852 velopment Capabilities Association Conference'. 1853

Martens, K. \& Golub, A. (2012), '11. a justice- 1854 theoretic exploration of accessibility measures', 1855 Accessibility analysis and transport planning: 1856 Challenges for Europe and North America 195. 1857

Miller, H. J. (2005), Place-based versus people- 1858 based accessibility, in 'Access to destinations', 1859 Emerald Group Publishing Limited, pp. 63-89. 1860

Mitra, S. (2006), 'The capability approach and 1861 disability', Journal of disability policy studies 1862 16(4), 236-247. 
Neutens, T., Schwanen, T., Witlox, F. \& De Maeyer, P. (2010), 'Equity of urban service delivery: a comparison of different accessibility measures', Environment and Planning A 42(7), 1613-1635.

Noble, M., McLennan, D., Wilkinson, K., Whitworth, A., Exley, S., Barnes, H., Dibben, C., McLennan, D. et al. (2007), 'The english indices of deprivation 2007'.

Nordbakke, S. (2013), 'Capabilities for mobility among urban older women: barriers, strategies and options', Journal of transport geography 26, 166-174.

Nutley, S. (1998), 'Rural areas: the accessibility problem', Modern Transport Geography, 2nd rev. ed., Wiley and sons, Chichester pp. 185-215.

Ordnance Survey (2012), 'Points of interest classification scheme'.

Orr, S. (2010), Evaluation of transport accessibility for elderly and disabled people: a proposal for an activity-based quality of life approach, in 'European Transport Conference, 2010Association for European Transport'.

Páez, A., Gertes Mercado, R., Farber, S., Morency, C. \& Roorda, M. (2010), 'Relative accessibility deprivation indicators for urban settings: definitions and application to food deserts in montreal', Urban Studies 47(7), 1415-1438.

Pardo, L. (2005), Statistical inference based on divergence measures, CRC Press.

Pelletier, M.-P., Trépanier, M. \& Morency, C. (2011), 'Smart card data use in public transit: A literature review', Transportation Research Part C: Emerging Technologies 19(4), 557-568.

Pereira, R. H., Schwanen, T. \& Banister, D. (2017), 'Distributive justice and equity in transportation', Transport Reviews 37(2), 170-191.

Pirie, G. (1981), 'The possibility and potential of public policy on accessibility', Transportation Research Part A: General 15(5), 377-381.
Preston, J. \& Rajé, F. (2007), 'Accessibility, mobil- 1904 ity and transport-related social exclusion', Jour- 1905 nal of Transport Geography 15(3), 151-160. 1906

Pyrialakou, V. D., Gkritza, K. \& Fricker, J. D. 1907 (2016), 'Accessibility, mobility, and realized 1908 travel behavior: Assessing transport disadvan- 1909 tage from a policy perspective', Journal of trans- 1910 port geography 51, 252-269.

Rashid, K., Yigitcanlar, T. \& Bunker, J. M. 1912 (2010), 'Minimising transport disadvantage to 1913 support knowledge city formation: applying the 1914 capability approach to select indicators', Mel- 1915 bourne 2010 Knowledge Cities World Summit: 1916 3rd Knowledge Cities World Summit. .

1917

Rawls, J. (2009), A theory of justice, Harvard uni- 1918 versity press.

Robeyns, I. (2005), 'Selecting capabilities for qual- 1920 ity of life measurement', Social indicators re- 192 search 74(1), 191-215. 1922

Ryan, J., Wretstrand, A. \& Schmidt, S. M. ${ }_{1923}$ (2015), 'Exploring public transport as an ele- 1924 ment of older persons' mobility: A capability 1925 approach perspective', Journal of transport ge- 1926 ography 48, 105-114.

1927

Schönfelder, S. \& Axhausen, K. W. (2003), 'Activ- 1928 ity spaces: measures of social exclusion?', Trans- 1929 port policy 10(4), 273-286. 1930

Sen, A. (2014), 'Development as freedom (1999)', 1931 Roberts, JT, Hite, AB \& Chorev, N. The Glob- 1932 alization and Development Reader: Perspectives 1933 on Development and Global Change 2, 525-547. 1934

Sen, A. et al. (1990), 'Development as capability 1935 expansion', Human development and the inter- 1936 national development strategy for the 1990s $\mathbf{1} . \quad{ }_{1937}$

Smith, N., Hirsch, D. \& Davis, A. (2012), 'Acces- 1938 sibility and capability: the minimum transport 1939 needs and costs of rural households', Journal of 1940 Transport Geography 21, 93-101.

194

Social Exclusion Unit (2003), 'Making the connec- 1942 tions: final report on transport and social exclu- 1943 sion', http://webarchive. nationalarchives. gov. 1944 $u k$. 
Titheridge, H., Achuthan, K., Mackett, R. \& Solomon, J. (2009), 'Assessing the extent of transport social exclusion among the elderly'.

Titheridge, H., Mackett, R., Christie, N., Oviedo Hernández, D. \& Ye, R. (2014), 'Transport and poverty: a review of the evidence'.

Transport for London (2011), 'Travel in london,supplementary report:london travel demandsurvey (ltds)'. Online; Accessed 29/03/2016.

URL: http://content.tfl.gov.uk/london-traveldemand-survey.pdf

Tyler, N. (2006), 'Capabilities and radicalism: Engineering accessibility in the 21st century', Transportation planning and technology 29(5), 331-358.

Van Wee, B. \& Geurs, K. (2011), 'Discussing equity and social exclusion in accessibility evaluations', EJTIR 11(4), 350-367.

Wu, B. M. \& Hine, J. P. (2003), 'A ptal approach to measuring changes in bus service accessibility', Transport Policy 10(4), 307-320.

Yang, X. \& Day, J. (2016), Operationalizing the capabilities approach for urban policy evaluation: The travel welfare impacts of government job resettlement, in 'Geography Research Forum', Vol. 35, pp. 113-137. 
A Posterior activity distributions for the individuals of the uncon- ${ }_{1973}$ strained, $>60$ and low income population groups

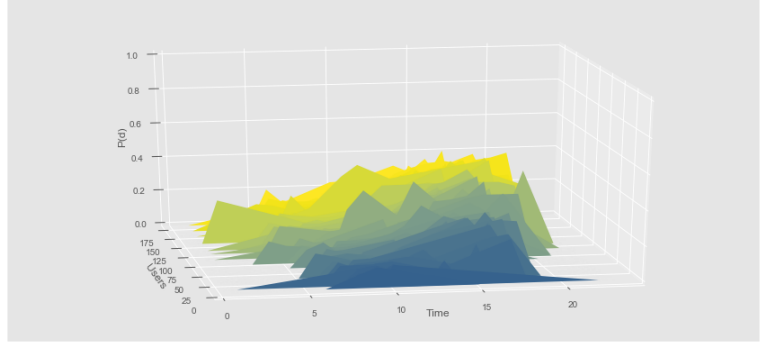

(a) Eating and drinking

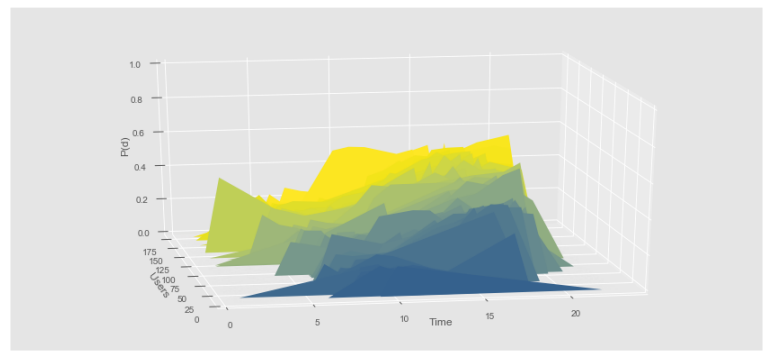

(c) Retail

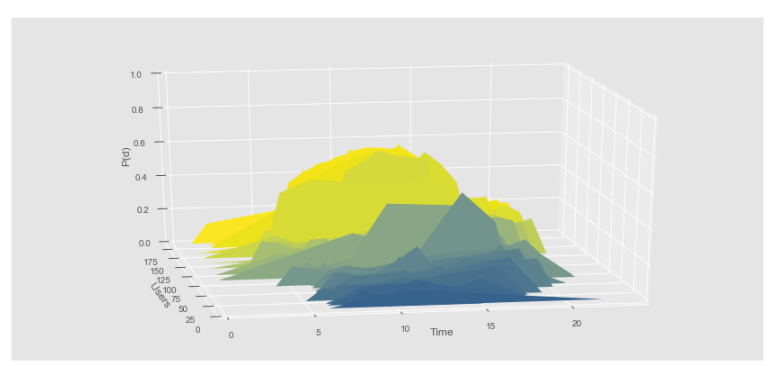

(b) Education and health

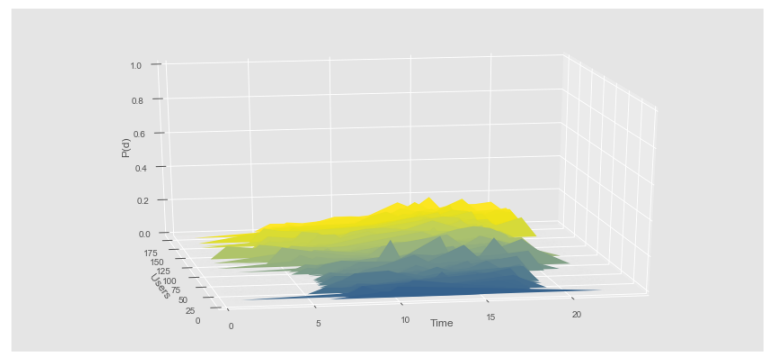

(d) Outdoors and Recreation

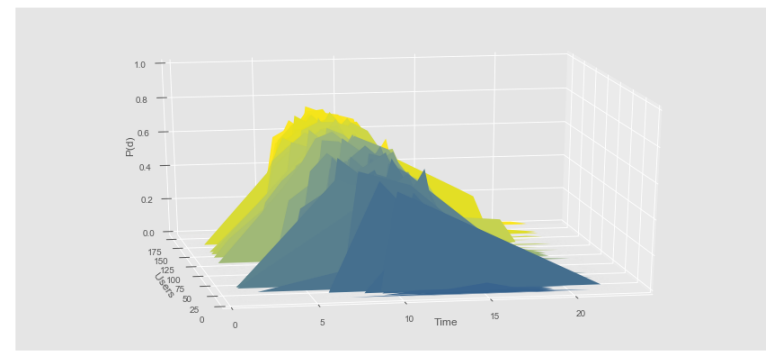

(e) Employment

Figure 11: Posterior distributions of activity types for the unconstrained population sample 


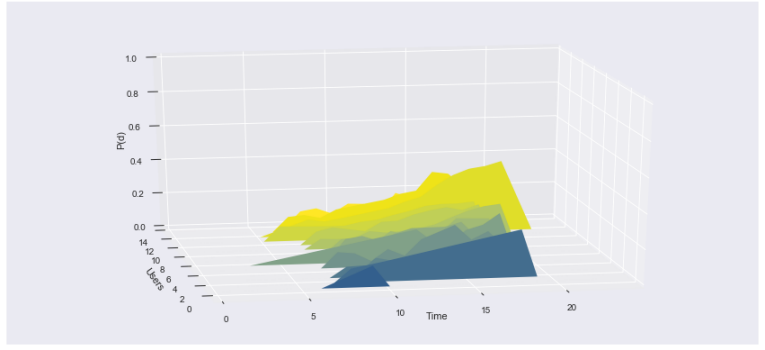

(a) Eating and drinking

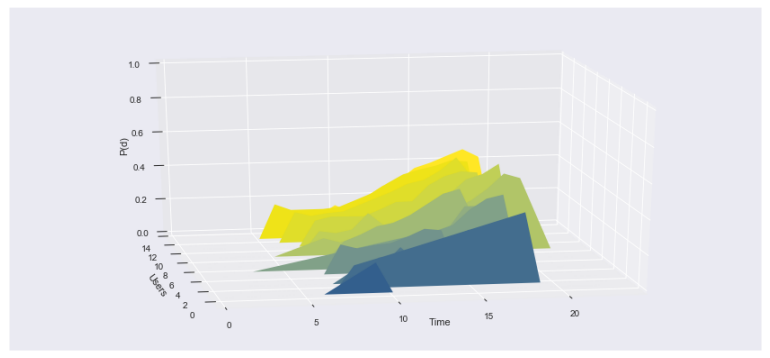

(c) Retail

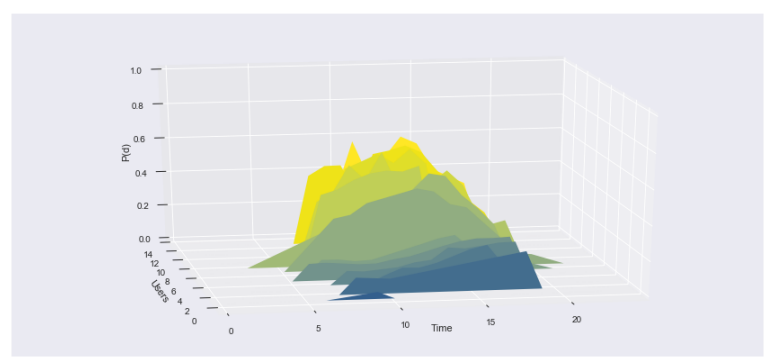

(b) Education and health

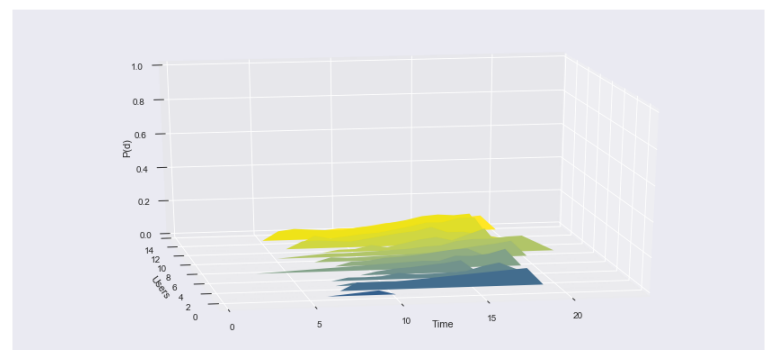

(d) Outdoors and Recreation

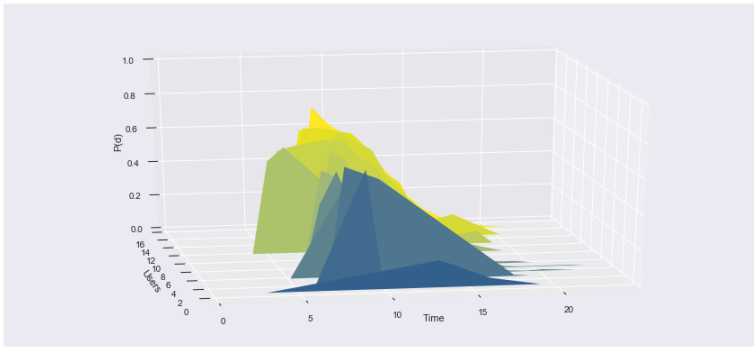

(e) Employment

Figure 12: Posterior distributions of activity types for the low income population sample 


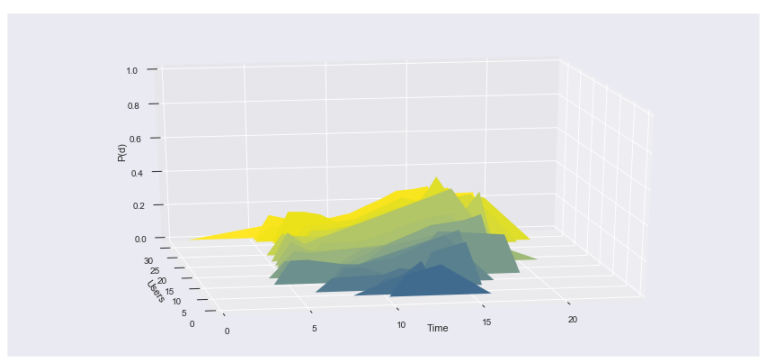

(a) Eating and drinking

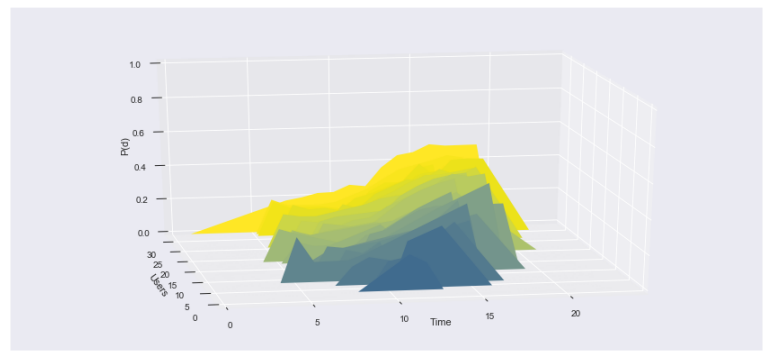

(c) Retail

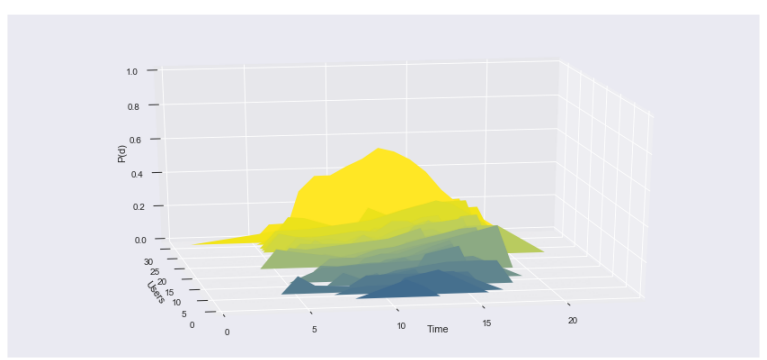

(b) Education and health

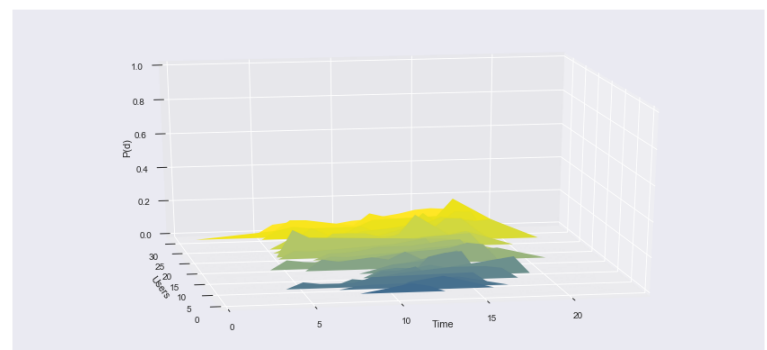

(d) Outdoors and Recreation

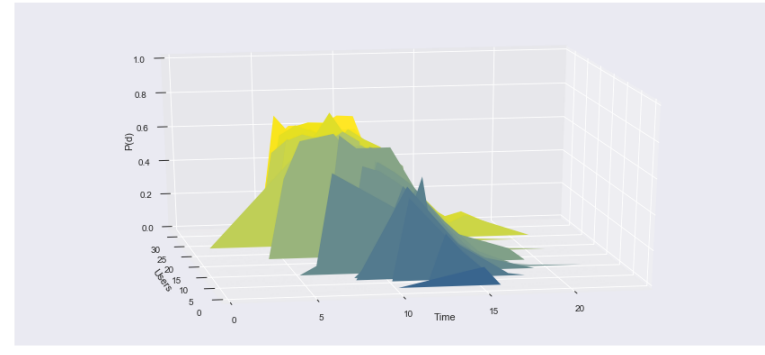

(e) Employment

Figure 13: Posterior distributions of activity types for the $>60$ years old population sample 


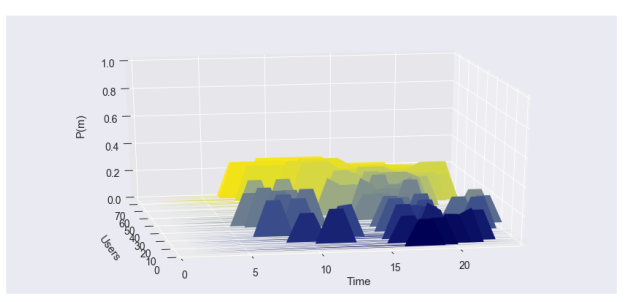

(a) Posterior means Bus/Eating and Drinking (b) Posterior means Rail/Eating and Drinking (c) Posterior means Tram/Eating and Drinking
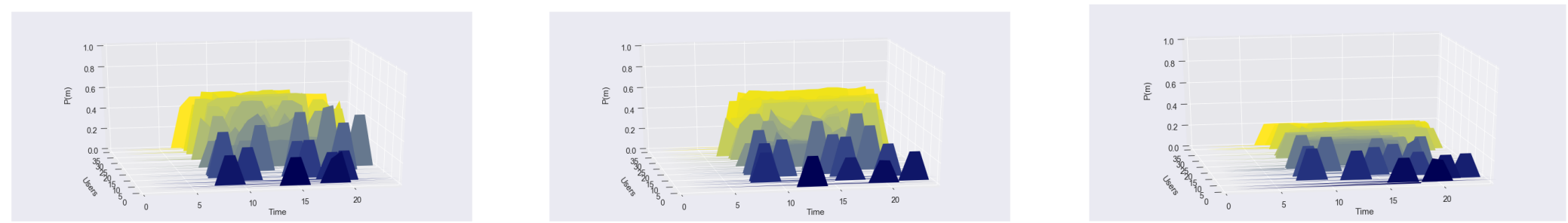

(d) Posterior means Bus/Education and Health(e) Posterior means Rail/Education and Health (f) Posterior means Tram/Education and Health

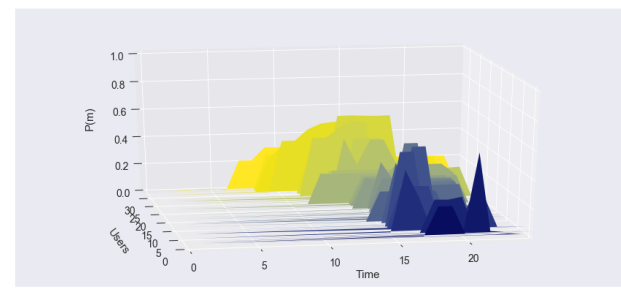

(g) Posterior means Bus/Retail

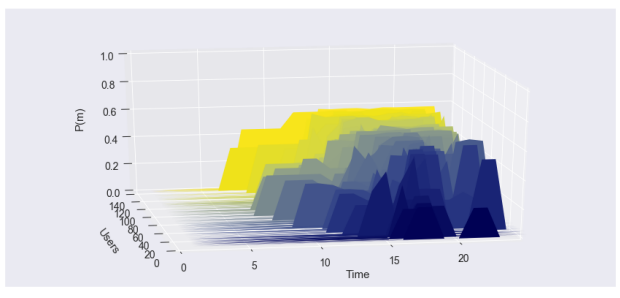

(h) Posterior means Rail/Retail

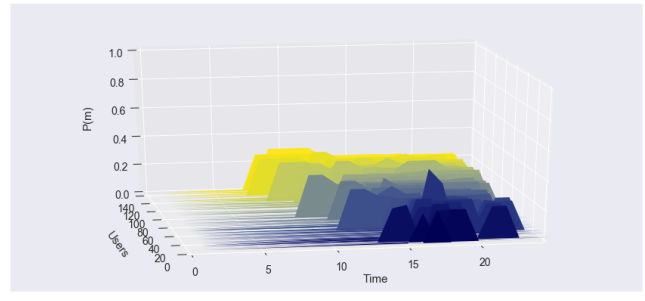

(i) Posterior means Tram/Retail 

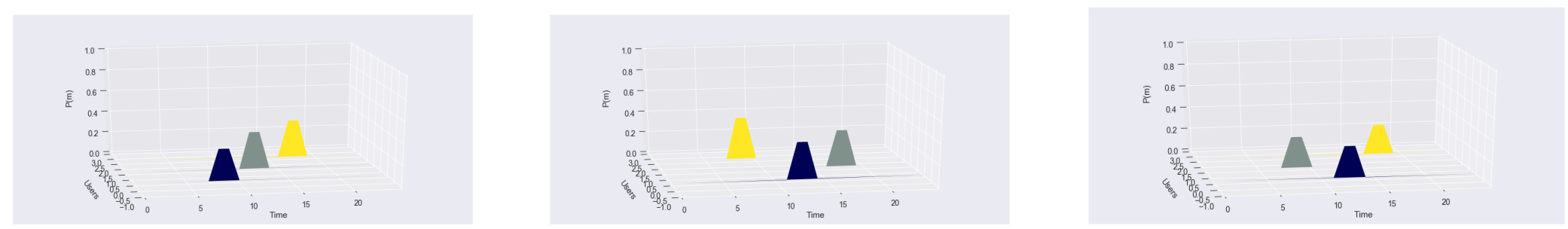

(j) Posterior means Bus/Outdoors and Recre-(k) Posterior means Rail/Outdoors and Recre- (l) Posterior means Tram/Outdoors and Recreation ation ation

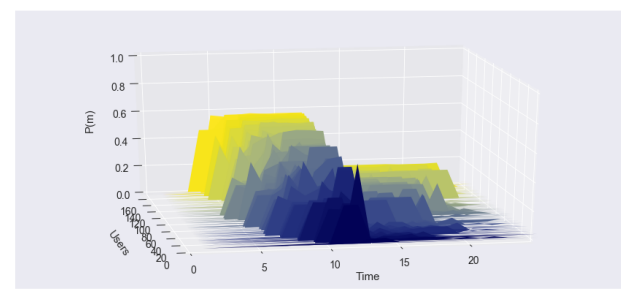

(m) Posterior means Bus/Employment

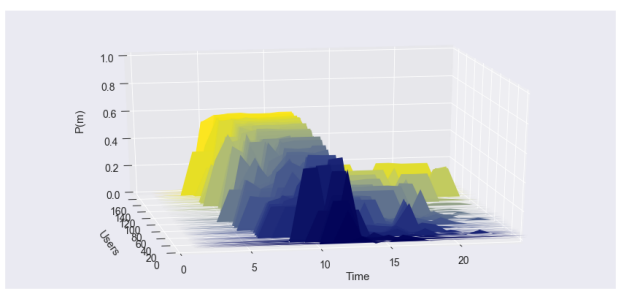

(n) Posterior means Rail/Employment

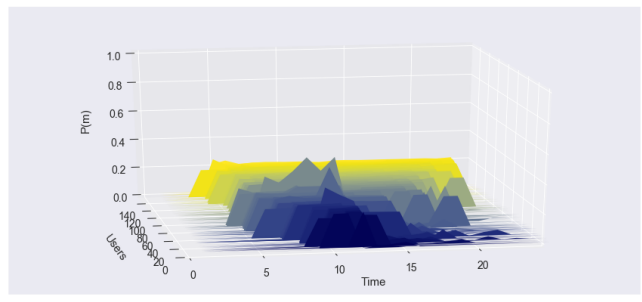

(o) Posterior means Tram/Employment

Figure 14: Posterior means for the unconstrained population group 

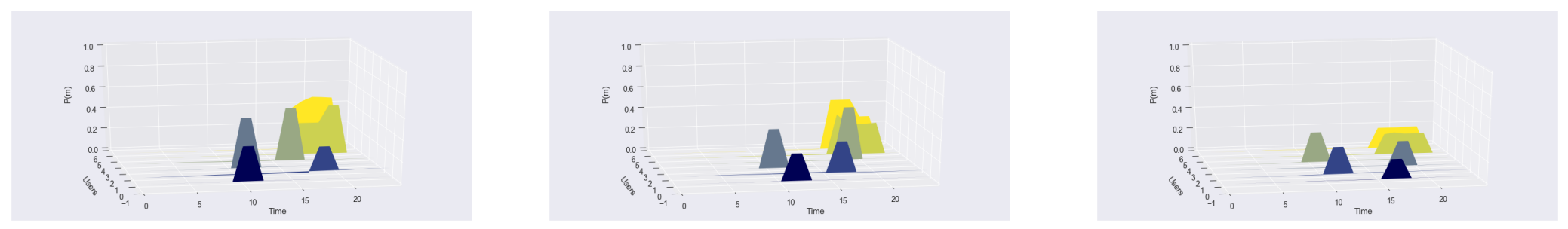

(a) Posterior means Bus/Eating and Drinking (b) Posterior means Rail/Eating and Drinking (c) Posterior means Tram/Eating and Drinking
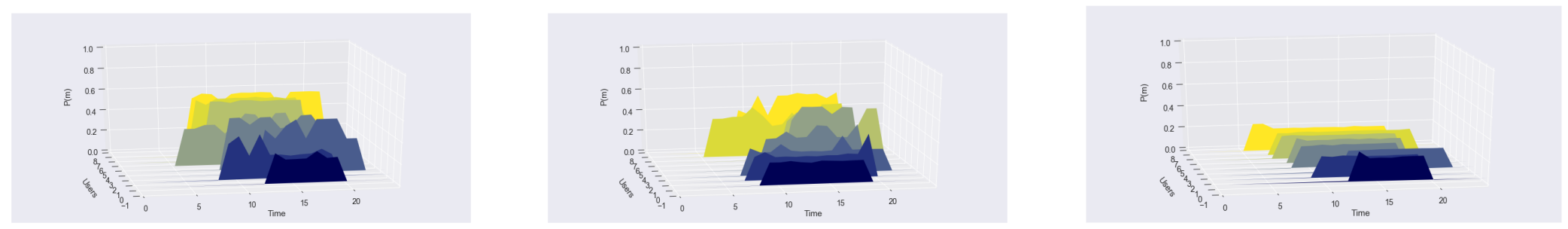

(d) Posterior means Bus/Education and Health(e) Posterior means Rail/Education and Health (f) Posterior means Tram/Education and

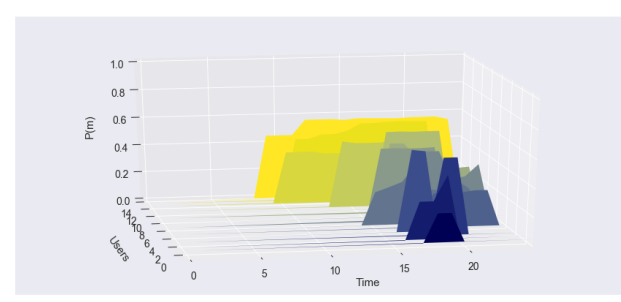

(g) Posterior means Bus/Retail

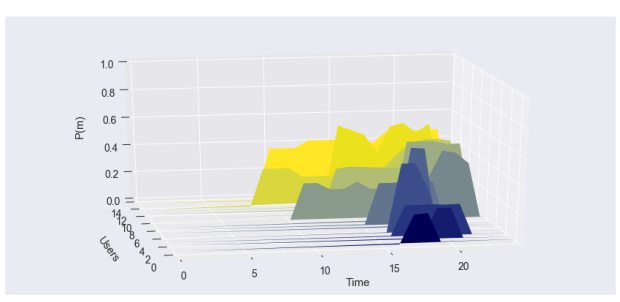

(h) Posterior means Rail/Retail Health

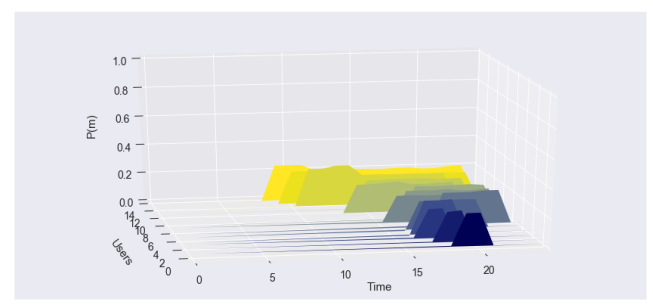

(i) Posterior means Tram/Retail 

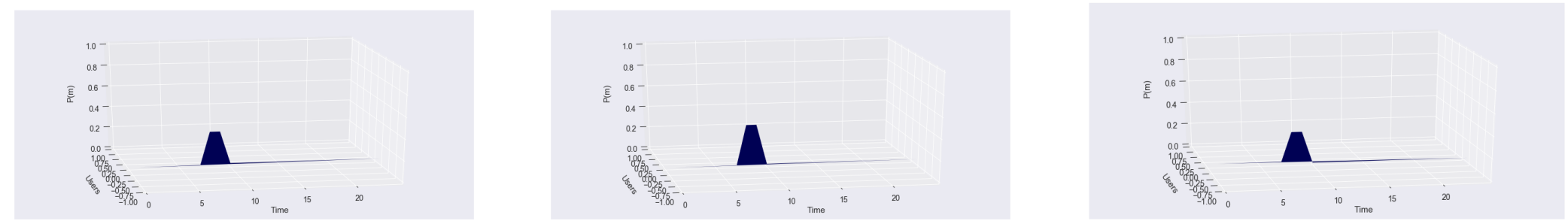

(j) Posterior means Bus/Outdoors and Recre-(k) Posterior means Rail/Outdoors and Recre- (l) Posterior means Tram/Outdoors and Recreation ation ation

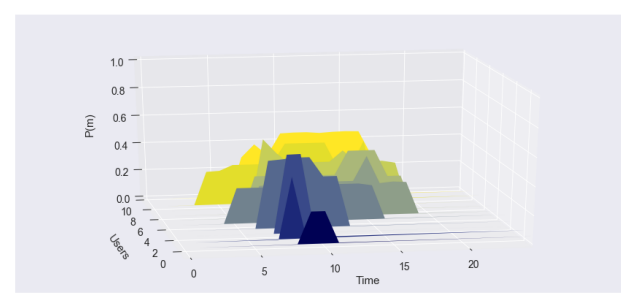

(m) Posterior means Bus/Employment

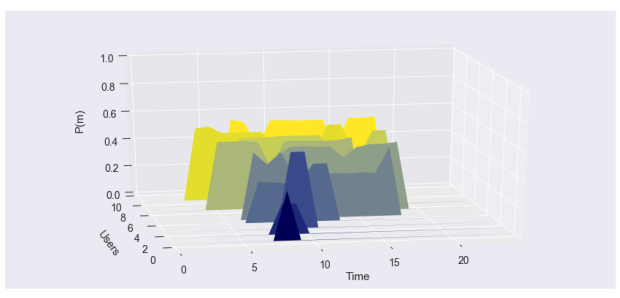

(n) Posterior means Rail/Employment

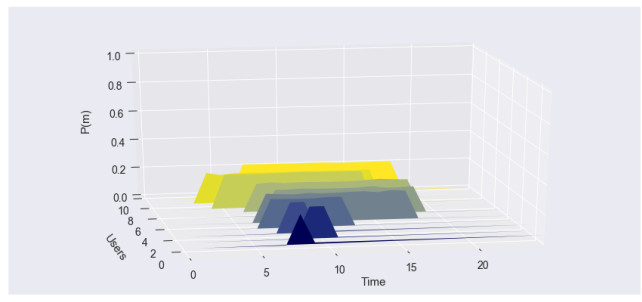

(o) Posterior means Tram/Employment

Figure 15: Posterior means for the low income population group 

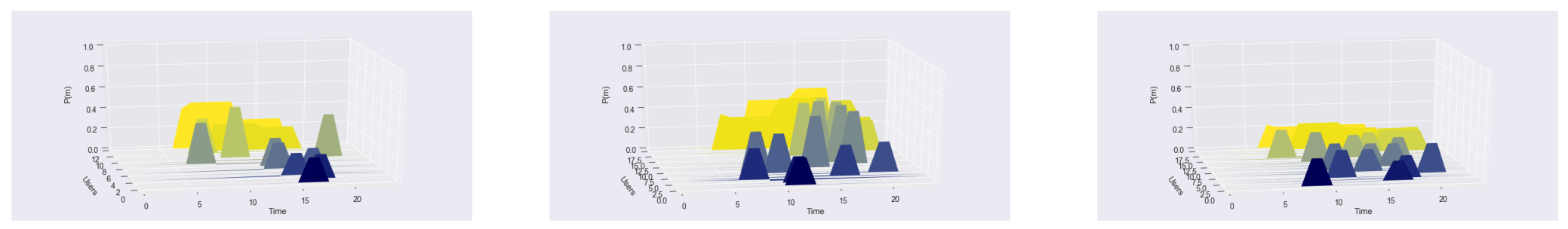

(a) Posterior means Bus/Eating and Drinking (b) Posterior means Rail/Eating and Drinking (c) Posterior means Tram/Eating and Drinking
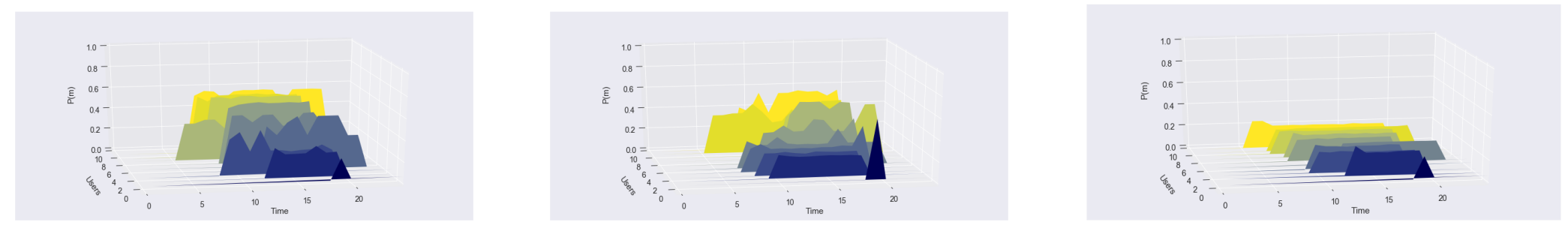

(d) Posterior means Bus/Education and Health(e) Posterior means Rail/Education and Health (f) Posterior means Tram/Education and

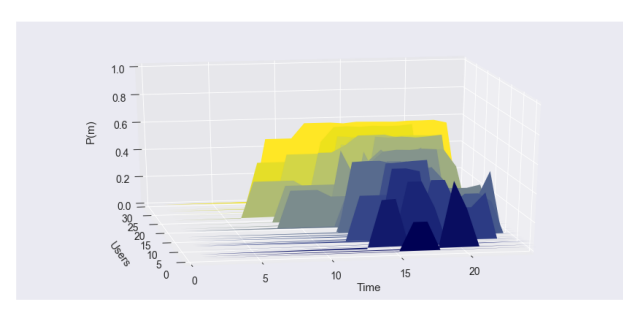

(g) Posterior means Bus/Retail

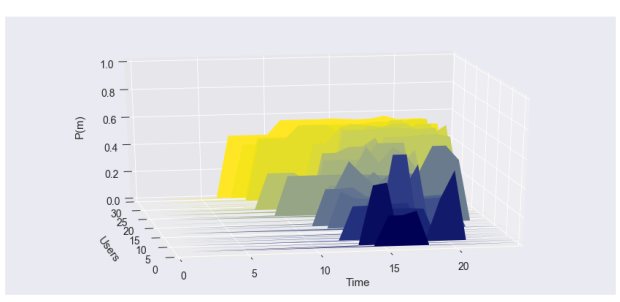

(h) Posterior means Rail/Retail Health

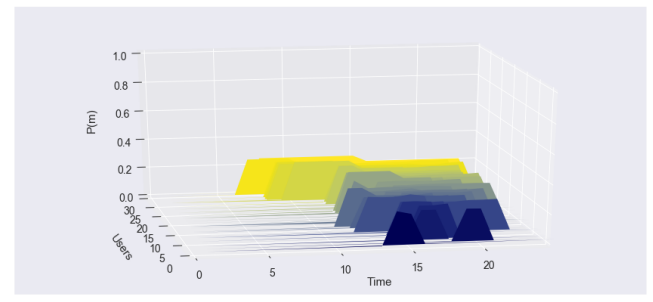

(i) Posterior means Tram/Retail 

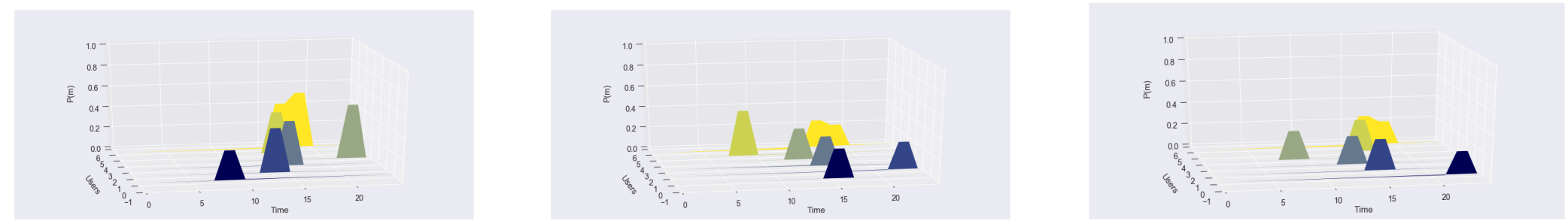

(j) Posterior means Bus/Outdoors and Recre-(k) Posterior means Rail/Outdoors and Recre- (l) Posterior means Tram/Outdoors and Recreation ation ation

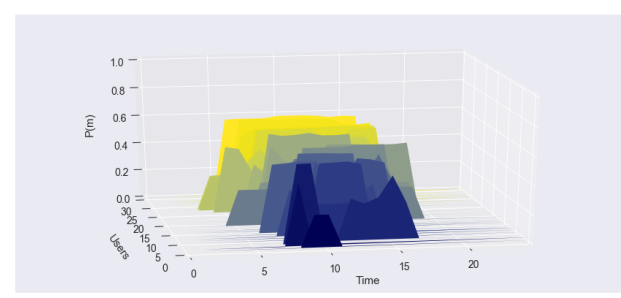

(m) Posterior means Bus/Employment

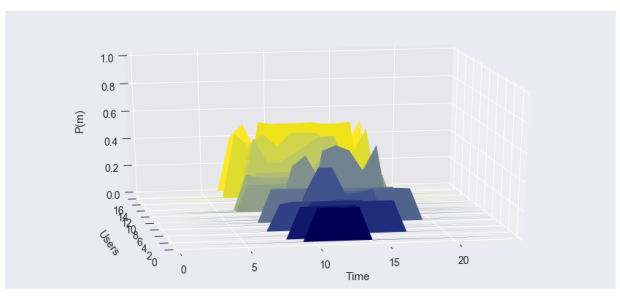

(n) Posterior means Rail/Employment

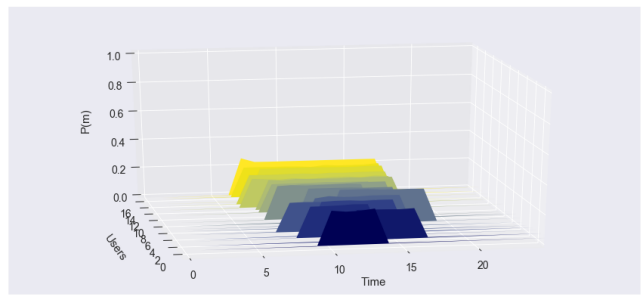

(o) Posterior means Tram/Employment

Figure 16: Posterior means for the ¿ 60 population group 


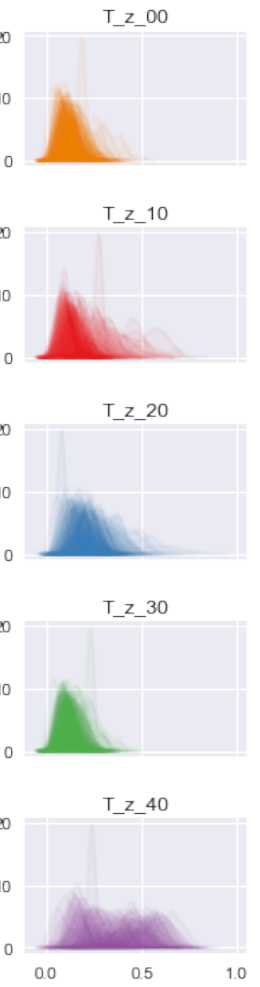

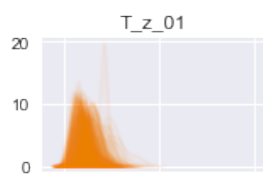
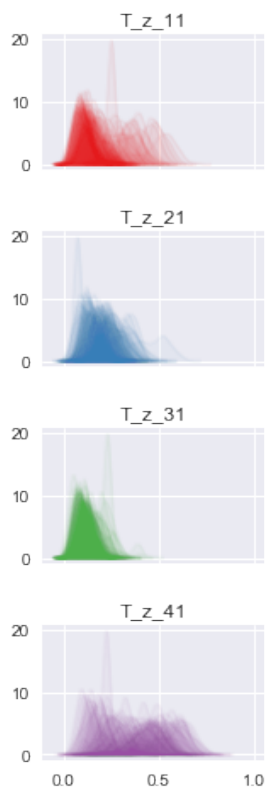
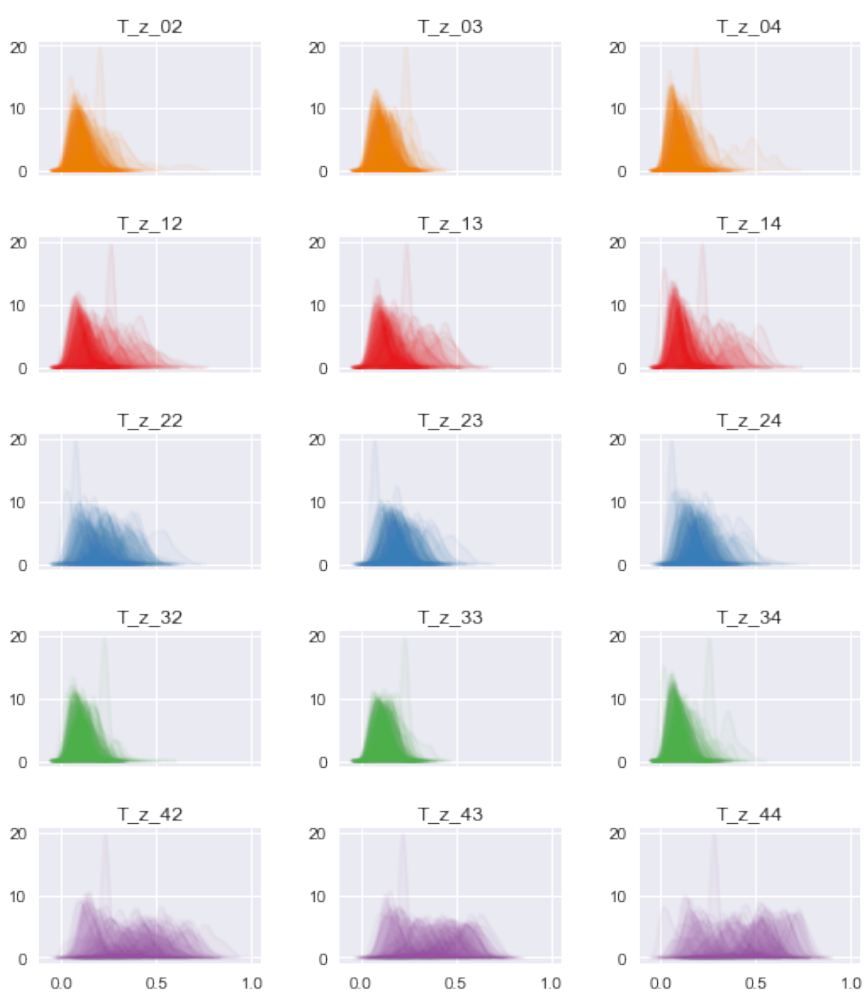

Transition probabilities

Figure 17: Posterior densities for $T_{z}$ 

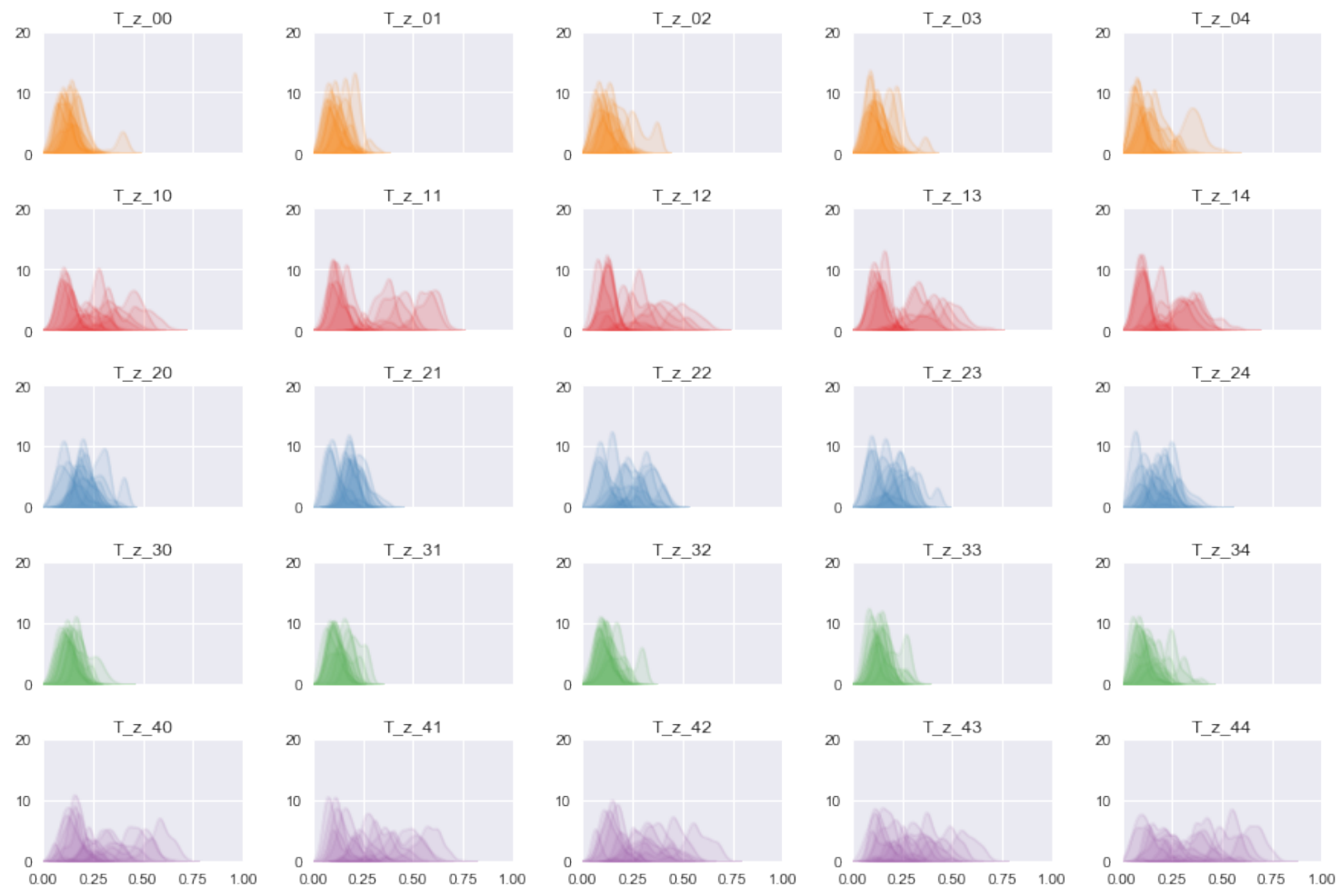

\section{Transition probabilities}

Figure 18: Posterior densities for $T_{z}$ for the low income population group 

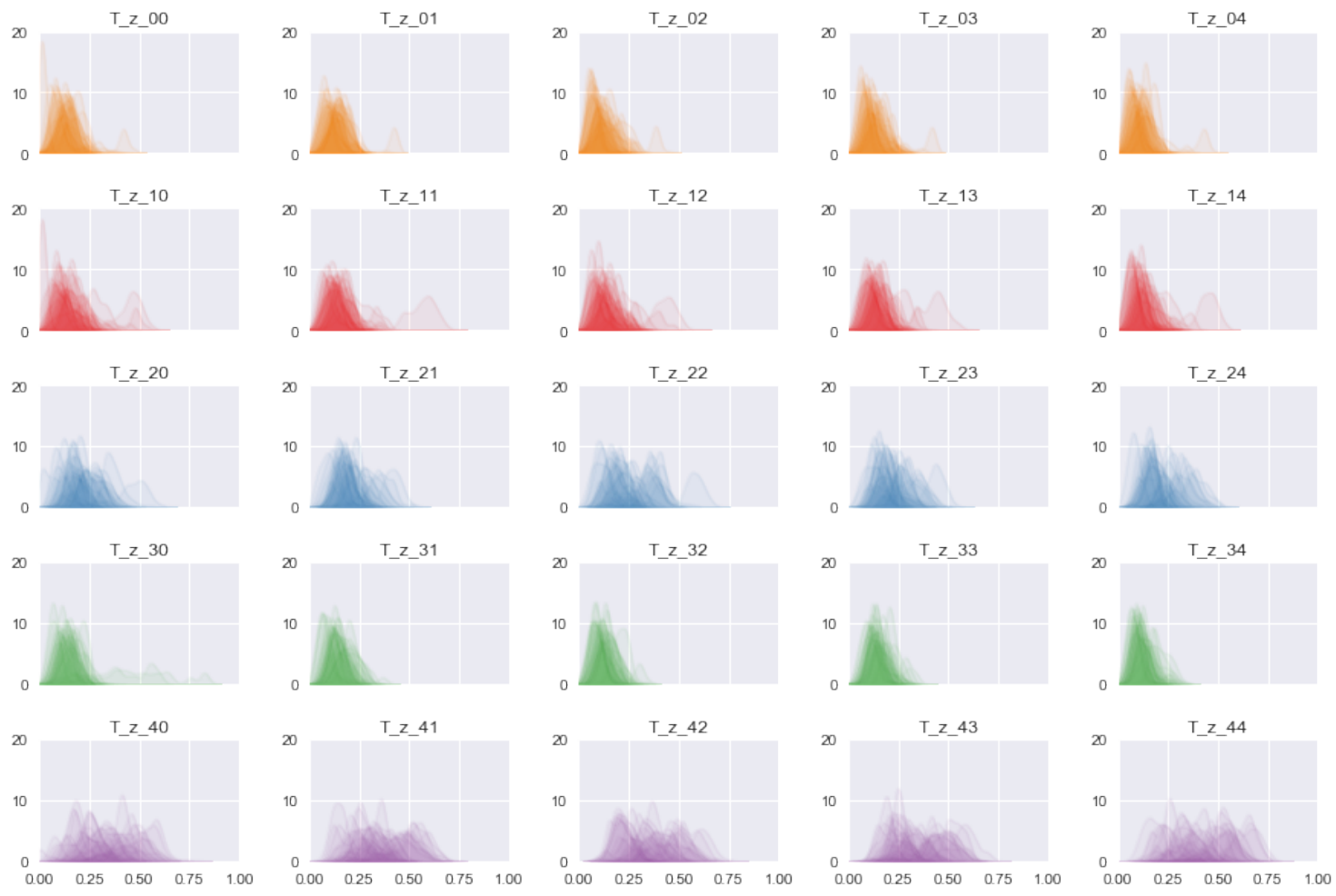

\section{Transition probabilities}

Figure 19: Posterior densities for $T_{z}$ for the over sixty population group 

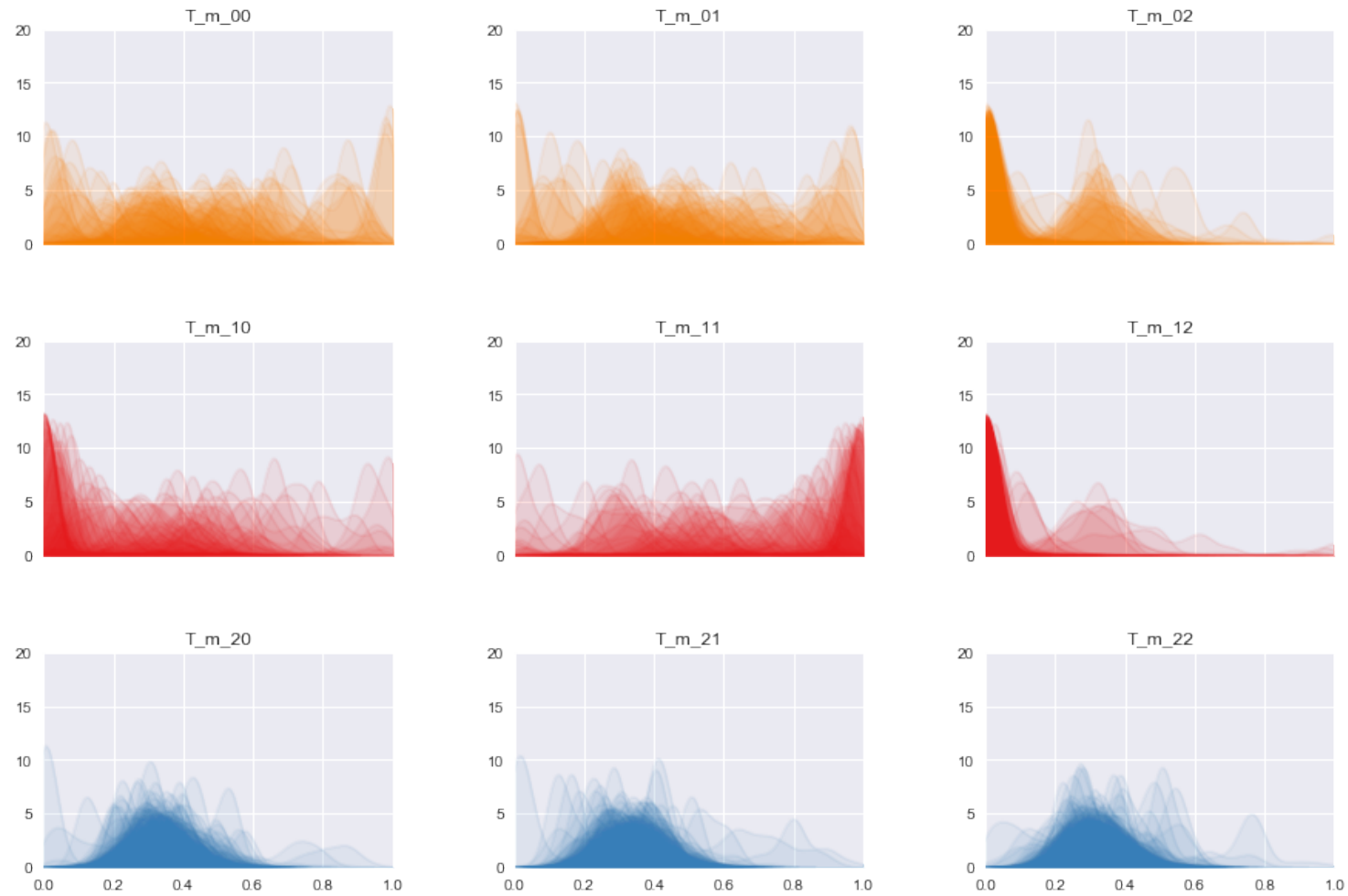

Transition probabilities

Figure 20: Posterior densities for $T_{m}$ for the unconstrained population group 

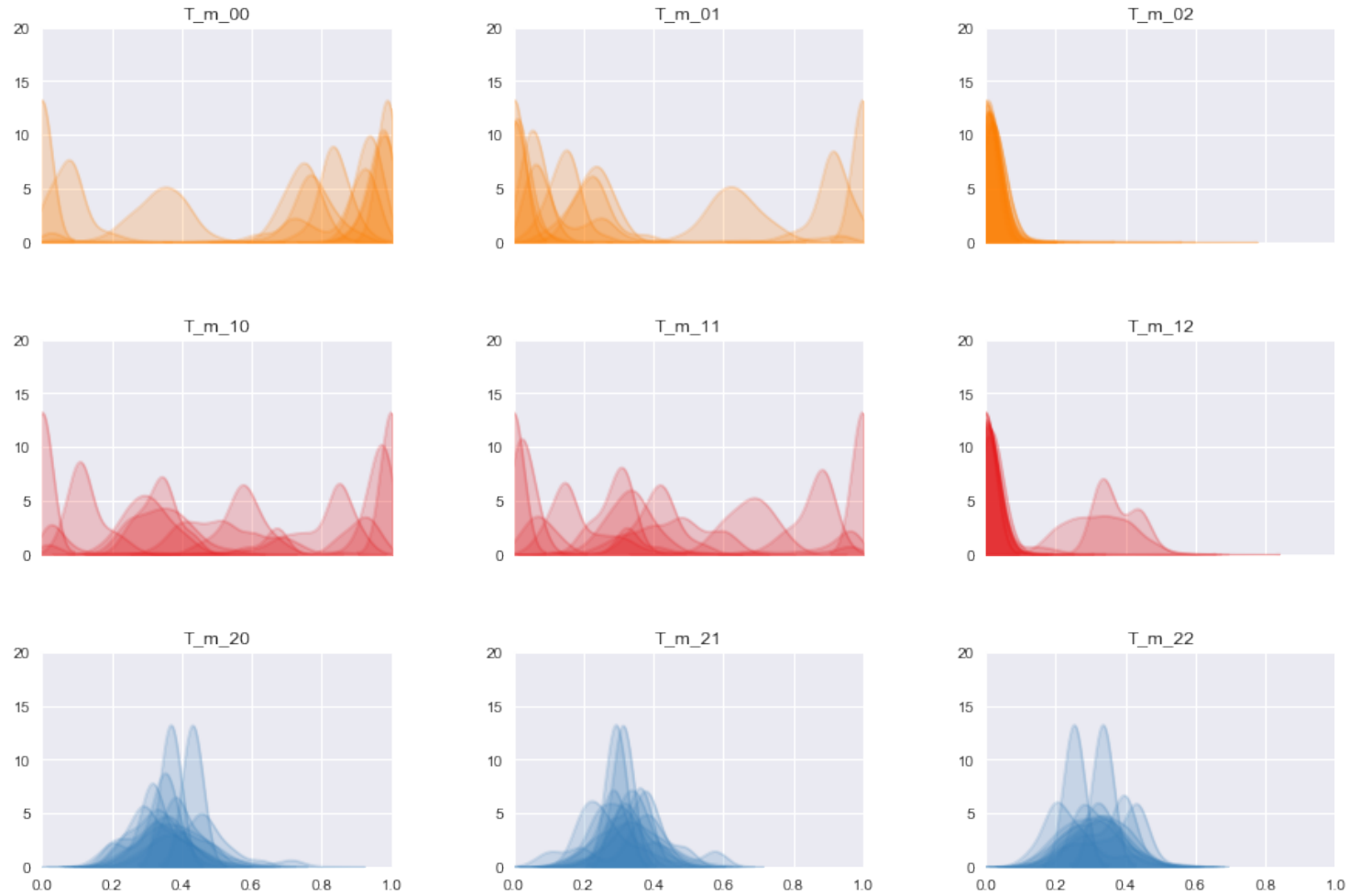

Transition probabilities

Figure 21: Posterior densities for $T_{m}$ for the over sixty population group 

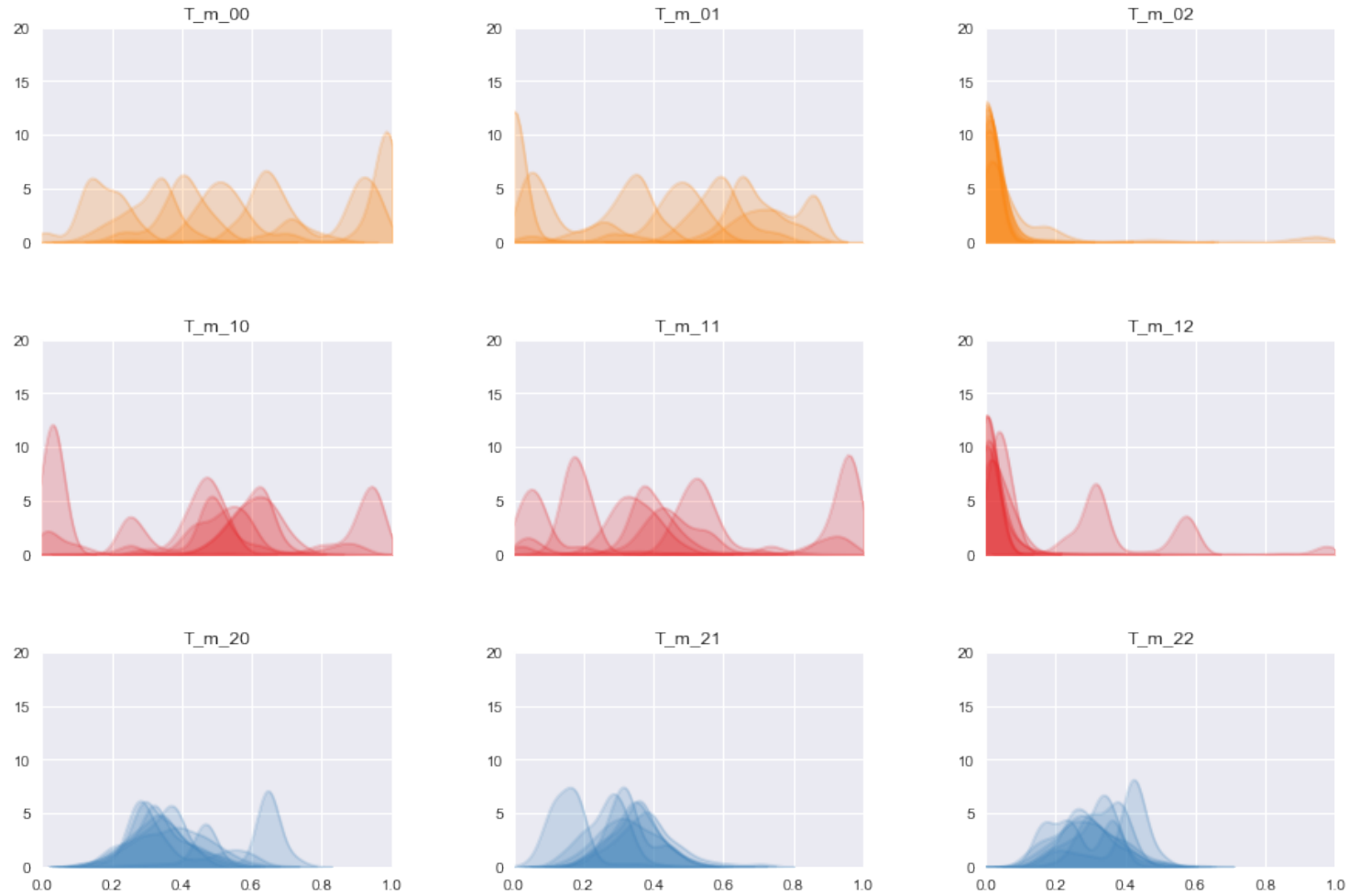

\section{Transition probabilities}

Figure 22: Posterior densities for $T_{m}$ for the low income population group 